\title{
Design of CDTA and VDTA Based Frequency Agile Filters
}

\author{
Neeta Pandey, ${ }^{1}$ Aseem Sayal, ${ }^{2}$ Richa Choudhary, ${ }^{2}$ and Rajeshwari Pandey ${ }^{1}$ \\ ${ }^{1}$ Department of Electronics and Communication Engineering, Delhi Technological University, Delhi 110042, India \\ ${ }^{2}$ Department of Electrical Engineering, Delhi Technological University, Delhi 110042, India \\ Correspondence should be addressed to Neeta Pandey; n66pandey@rediffmail.com
}

Received 21 May 2014; Revised 3 November 2014; Accepted 3 November 2014; Published 23 December 2014

Academic Editor: Weisheng Zhao

Copyright (C) 2014 Neeta Pandey et al. This is an open access article distributed under the Creative Commons Attribution License, which permits unrestricted use, distribution, and reproduction in any medium, provided the original work is properly cited.

\begin{abstract}
This paper presents frequency agile filters based on current difference transconductance amplifier (CDTA) and voltage difference transconductance amplifier (VDTA). The proposed agile filter configurations employ grounded passive components and hence are suitable for integration. Extensive SPICE simulations using $0.25 \mu \mathrm{m}$ TSMC CMOS technology model parameters are carried out for functional verification. The proposed configurations are compared in terms of performance parameters such as power dissipation, signal to noise ratio (SNR), and maximum output noise voltage.
\end{abstract}

\section{Introduction}

The rapid evolution of wireless services has led to demand for one-fits-all "analog" front end solution. These services use different standards and therefore necessitate development of integrated multistandard transceivers as they result in reduction of size, price, complexity, and power consumption. The parameters of integrated transceiver can be modified in order to be able to adapt to the specifications of each standard [1]. Practically, the designs employ either elements handling various standards in parallel or reconfigurable elements. The frequency agile filter (FAF) [1-10] characterized by adjustment range, reconfigurability, and agility may be used in transceivers. The term shadow filters is sometimes used in literature to refer to FAF [11, 12]. The literature survey shows that a limited number of topologies of active FAF are available and are based on op-amp [1] and current mode active block $[2,3]$ and CMOS [4].

There is a wide range of current mode building blocks available in open literature. Among these blocks current difference transconductance amplifier (CDTA) [11] is most suitable for current mode signal processing owing to its low input and high output impedances, respectively. The VDTA is yet another recently introduced building block which works on a principle similar to that of CDTA except that the input current differencing unit is replaced by the voltage differencing circuit. Many applications such as filters and oscillators based on CDTA and VDTA are available and have been reported in the literature [13-27] and references cited therein.

The main intention of this paper is to present CDTA and VDTA based frequency agile filter topologies. The proposed filters are suitable for integration as these employ grounded capacitors and a resistor. The paper is organised as follows. The FAF implementation scheme is briefly reviewed in Section 2. The CDTA based Class 0 , Class 1, and Class 2 FAF are presented in Section 3. Section 4 deals with the realization of VDTA based Class 0 , Class 1 , and Class 2 FAF. In Section 5, nonideal analysis of filters is presented. Simulation results are provided in Section 6 to substantiate the proposed FAF topologies. The performance characteristics of filter topologies are described in Section 7. The paper is concluded in Section 8.

\section{Implementation Scheme of FAF}

The implementation scheme of frequency agile filter (FAF) [3] is briefly reviewed in this section.

2.1. Class O FAF. A classical second order filter with band pass $\left(I_{\mathrm{BP}}\right)$ and low pass $\left(I_{\mathrm{LP}}\right)$ outputs of Figure 1 is designated as 


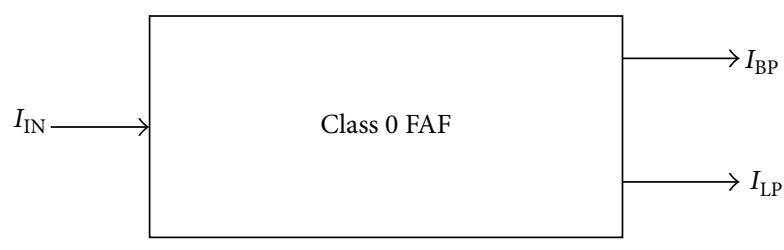

Figure 1: Class 0 FAF [3].

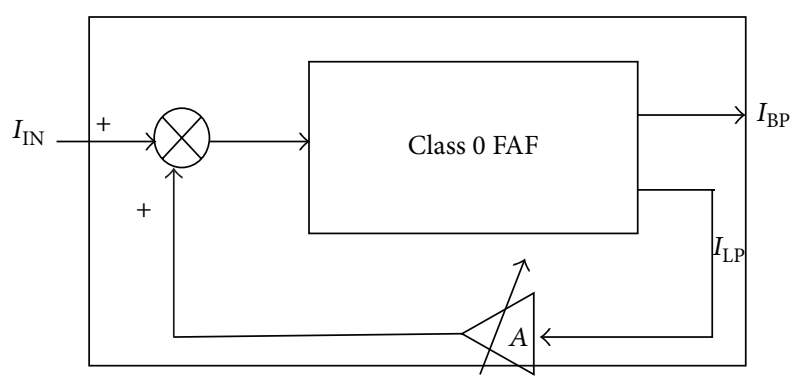

FIgure 2: Class 1 FAF.

Class 0 FAF [3]. The transfer functions of Class 0 FAF are given by

$$
\begin{aligned}
& T_{\mathrm{BP}}(s)=\frac{I_{\mathrm{BP}}}{I_{\mathrm{IN}}}=\frac{k s}{1+\alpha s+\beta s^{2}}, \\
& T_{\mathrm{LP}}(s)=\frac{I_{\mathrm{LP}}}{I_{\mathrm{IN}}}=\frac{p}{1+\alpha s+\beta s^{2}} .
\end{aligned}
$$

The center frequency $\left(f_{0}\right)$ and quality factor $(Q)$ of the filter are represented by (2) and (3), respectively:

$$
\begin{gathered}
f_{0}=\frac{1}{2 \pi \sqrt{\beta}}, \\
Q=\frac{\sqrt{\beta}}{\alpha} .
\end{gathered}
$$

2.2. Class 1 FAF. The basic block diagram of Class $1 \mathrm{FAF}$ is shown in Figure 2 wherein the low pass output of the Class 0 FAF is amplified (with variable gain $A$ ) and fed back to the input. The characteristic frequency $\left(f_{0 A}\right)$ and quality factor $\left(Q_{A}\right)$ of Class 1 FAF are given by $(4)$ and (5), respectively:

$$
\begin{aligned}
& f_{0 A}=f_{0} \sqrt{(1+A p)}, \\
& Q_{A}=Q \sqrt{(1+A p)} .
\end{aligned}
$$

2.3. Class $n$ FAF. The method outlined for Class 1 FAF realization can be extended for Class $n$ FAF implementation as shown in Figure 3. This requires $n$ amplifiers each with gain $A\left(A_{1}=A_{2}=\cdots=A_{n-1}=A_{n}\right)$ to be placed in $n$ feedback paths obtained in the same way as done in Class 1 implementation. The characteristic parameters of Class $n$ FAF are given by

$$
\begin{aligned}
& f_{0 A n}=f_{0}(1+A p)^{n / 2}, \\
& Q_{A n}=Q(1+A p)^{n / 2} .
\end{aligned}
$$

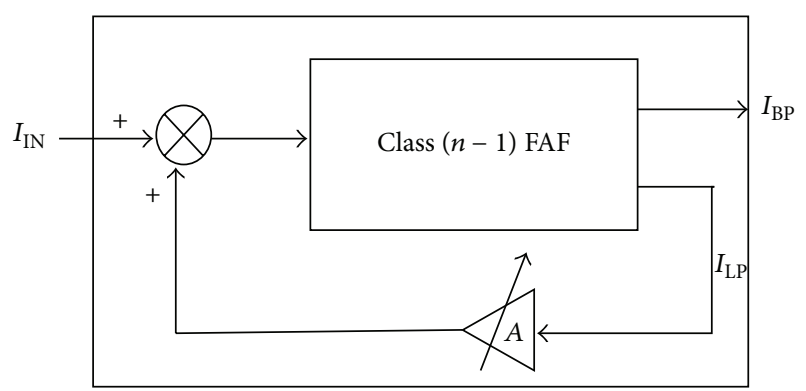

Figure 3: Class $n$ FAF.

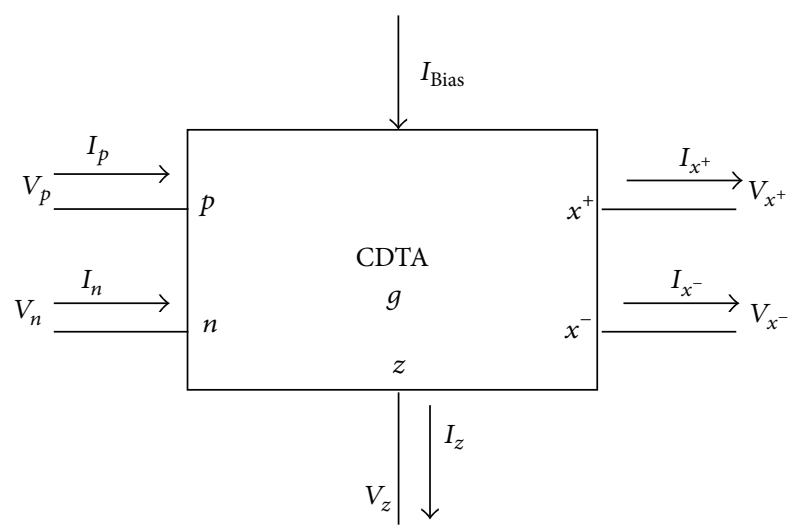

FIgURE 4: Symbol of CDTA.

\section{CDTA Based FAF}

The CDTA [11-18] consists of a unity-gain current source controlled by the difference of the input currents and a transconductance amplifier providing electronic tunability through its transconductance gain. The CDTA symbol is shown in Figure 4 and its terminal characteristics in matrix form are given by

$$
\left[\begin{array}{c}
V_{p} \\
V_{n} \\
I_{z} \\
I_{x+} \\
I_{x-}
\end{array}\right]=\left[\begin{array}{ccccc}
0 & 0 & 0 & 0 & 0 \\
0 & 0 & 0 & 0 & 0 \\
1 & -1 & 0 & 0 & 0 \\
0 & 0 & g & 0 & 0 \\
0 & 0 & g & 0 & 0
\end{array}\right]\left[\begin{array}{c}
I_{p} \\
I_{n} \\
V_{z} \\
V_{x+} \\
V_{x-}
\end{array}\right],
$$

where $g$ is transconductance of the CDTA. The CMOS implementation of CDTA [16] is given in Figure 5. The transistor network comprising transistors $\mathrm{Mcl}-\mathrm{Mcl}$ performs [16] current differencing operation on the currents entering at $p$ and $n$ nodes, which is available at $Z$ terminal. The voltage of $z$ terminal drives the source coupled pair (transistors (Mc18Mc21)) [16] of differential amplifier (Mc18-Mc26) giving a transconductance of $g$. The value of transconductance $(g)$ is expressed as

$$
g=\sqrt{2 \mu C_{o x}\left(\frac{W}{L}\right)_{19,21} I_{\text {Bias }}}
$$

which can be adjusted by bias current $I_{\text {Bias }}$ of CDTA. 


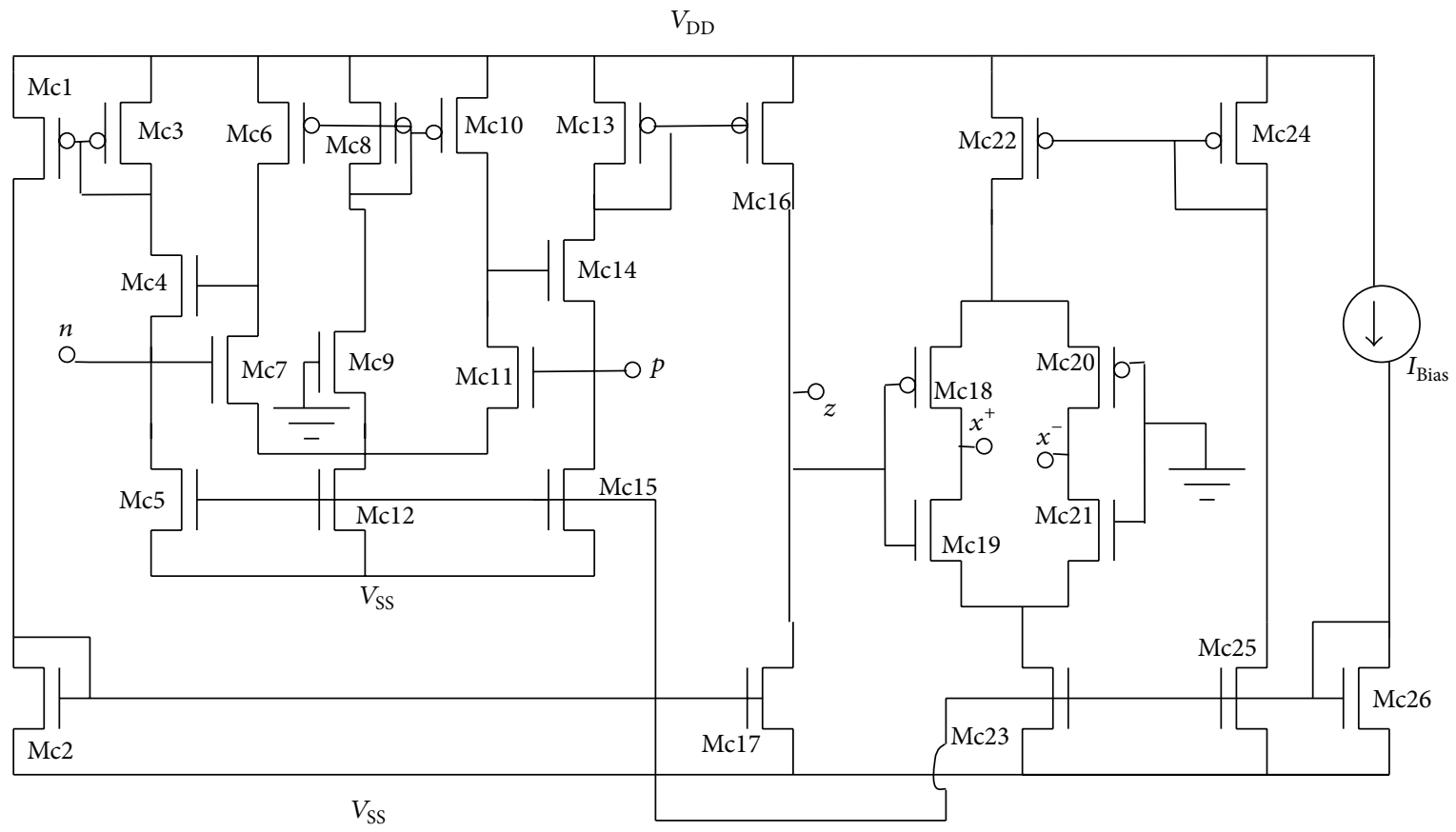

FIgURE 5: CMOS implementation of CDTA [16].

3.1. CDTA Based Class O FAF. The CDTA based second order filter employing two CDTA blocks and two grounded capacitors is shown in Figure 6. The second CDTA block uses additional TA block with its current output terminals denoted by $x_{c}^{+}$and $x_{c}^{-}$. It provides both low pass and band pass responses at high output impedance and can be used as Class 0 FAF. The current flowing through $x^{+}$and $x^{-}$ is controlled through transconductance $g_{2}$ whereas current flowing through terminals $x_{c}^{+}$and $x_{c}^{-}$is controlled through $g_{3}$. The low pass and band pass transfer functions of CDTA based Class 0 FAF are given by (9) and (10), respectively:

$$
\begin{aligned}
& \frac{I_{\mathrm{LP}}}{I_{\mathrm{IN}}}=\frac{g_{1} g_{2}}{C_{1} C_{2} s^{2}+s C_{1} g_{3}+g_{1} g_{2}}, \\
& \frac{I_{\mathrm{BP}}}{I_{\mathrm{IN}}}=\frac{s C_{2} g_{1}}{C_{1} C_{2} s^{2}+s C_{1} g_{3}+g_{1} g_{2}} .
\end{aligned}
$$

The center frequency and quality factor of Class $0 \mathrm{FAF}$ are expressed as

$$
\begin{gathered}
f_{0}=\frac{1}{2 \pi} \sqrt{\frac{g_{1} g_{2}}{C_{1} C_{2}}}, \\
Q=\frac{1}{g_{3}} \sqrt{\frac{g_{1} g_{2} C_{2}}{C_{1}}} .
\end{gathered}
$$

It may be noted that the $Q$ of the Class 0 FAF can be controlled independent of $f_{0}$ by varying $g_{3}$.

3.2. CDTA Based Class 1 FAF. The CDTA based Class 1 FAF is shown in Figure 7. It employs Class 0 FAF of Figure 6 along with an additional TA block (provides an output current which is product of its transconductance and voltage difference between noninverting $(+)$ and inverting $(-)$ terminals) and one grounded resistor. The TA block in the feedback path functions as an amplifier with tunable gain $A$ as given in

$$
A=g_{4} R,
$$

where $g_{4}$ is the transconductance of TA block and is given by $\sqrt{2 \mu C_{o x}(W / L)_{19,21} I_{\text {Bias } 4}}$.

The low pass and band pass transfer functions of CDTA based Class 1 FAF are given by (14) and (15), respectively:

$$
\begin{aligned}
& \frac{I_{\mathrm{LP}}}{I_{\mathrm{IN}}}=\frac{g_{1} g_{2}}{C_{1} C_{2} s^{2}+s C_{1} g_{3}+g_{1} g_{2}\left(1+R g_{4}\right)}, \\
& \frac{I_{\mathrm{BP}}}{I_{\mathrm{IN}}}=\frac{s g_{1} C_{2}}{C_{1} C_{2} s^{2}+s C_{1} g_{3}+g_{1} g_{2}\left(1+R g_{4}\right)} .
\end{aligned}
$$

The center frequency and quality factor of the CDTA based Class 1 FAF are expressed by (16) and (17), respectively:

$$
\begin{gathered}
f_{0 A}=\frac{1}{2 \pi} \sqrt{\frac{g_{1} g_{2}}{C_{1} C_{2}}} \sqrt{\left(1+g_{4} R\right),} \\
Q_{A}=\frac{1}{g_{3}} \sqrt{\frac{g_{1} g_{2} C_{2}}{C_{1}}} \sqrt{\left(1+g_{4} R\right) .}
\end{gathered}
$$

3.3. CDTA Based Class 2 FAF. The CDTA based Class 2 FAF is shown in Figure 8. It employs two CDTA blocks, two grounded capacitors, three TA blocks, and two grounded resistors. The TA blocks in the feedback path are used as amplifier with tunable gain $A$. The gain $A$ of TA based 


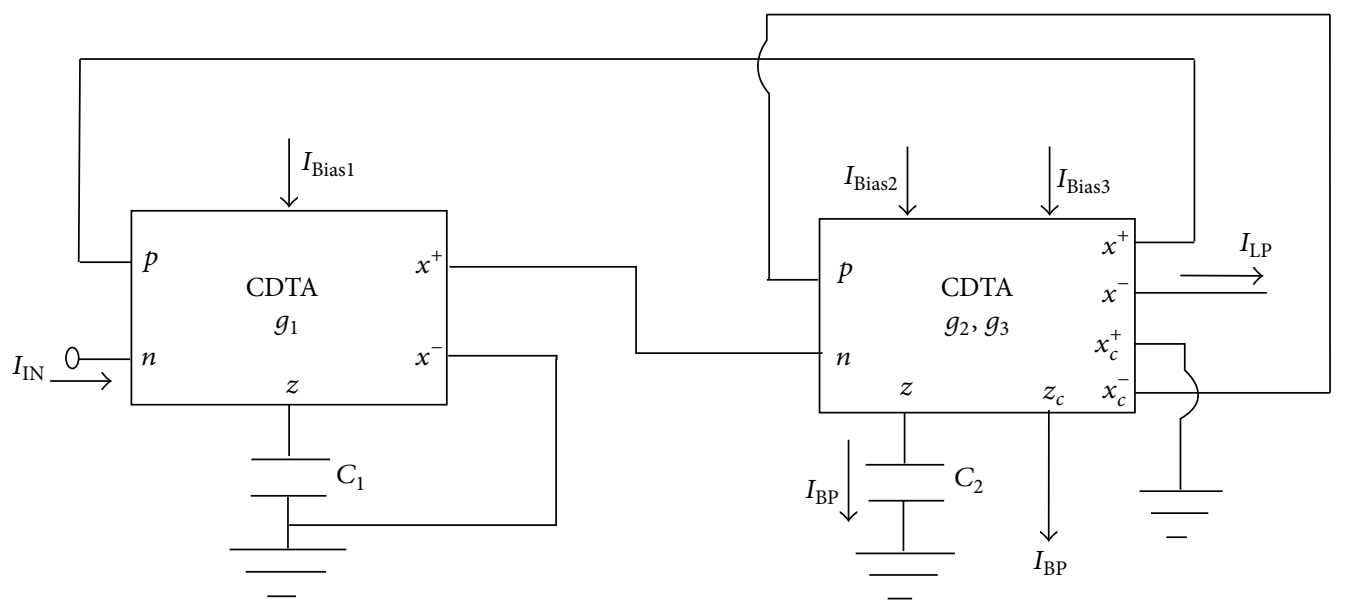

Figure 6: CDTA based Class 0 FAF.

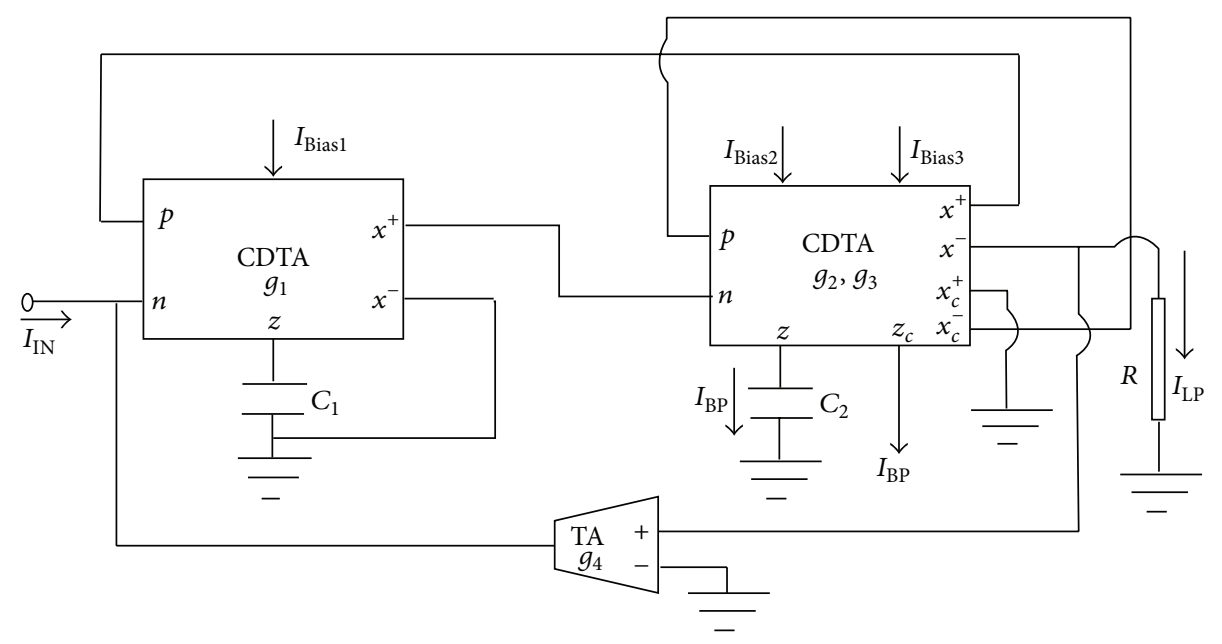

Figure 7: CDTA based Class 1 FAF.

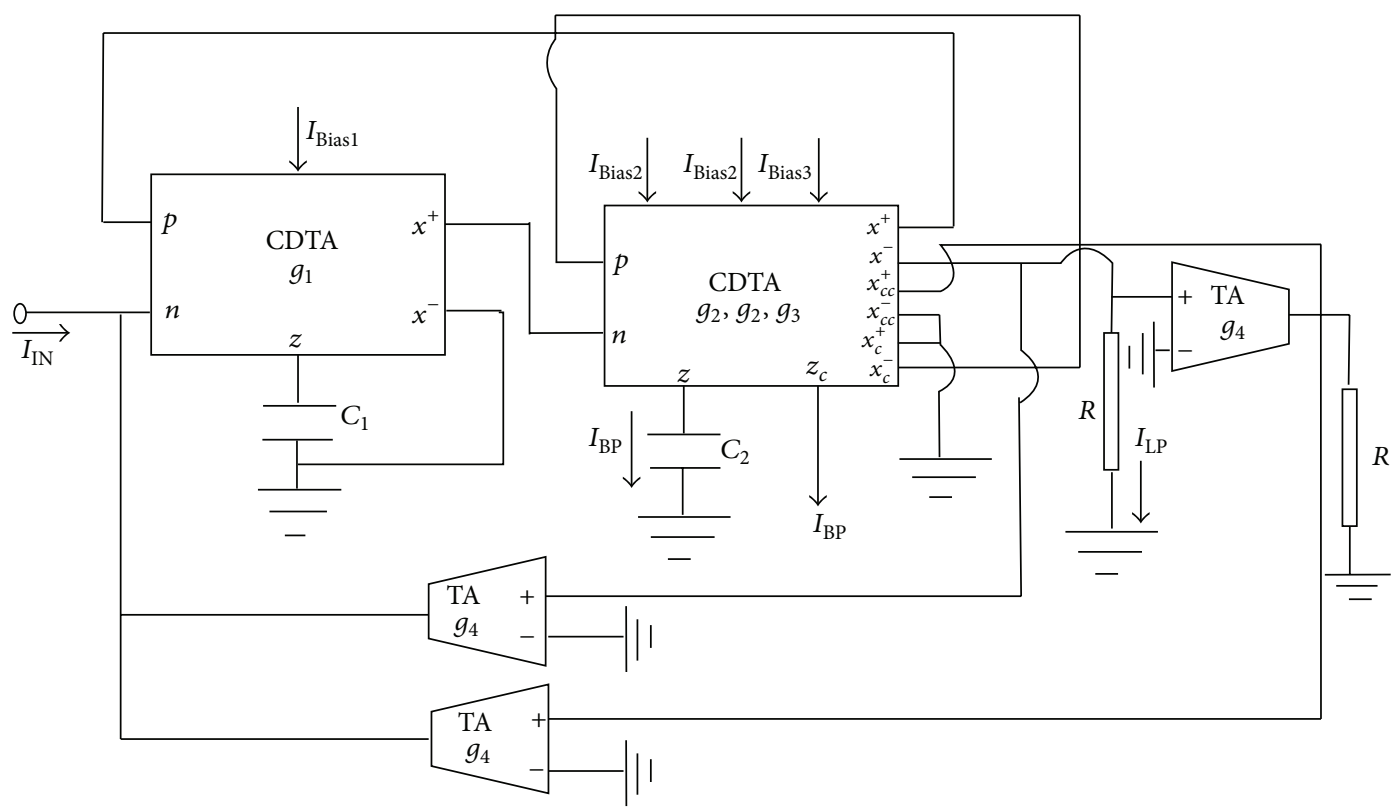

FIgURE 8: CDTA based Class 2 FAF. 


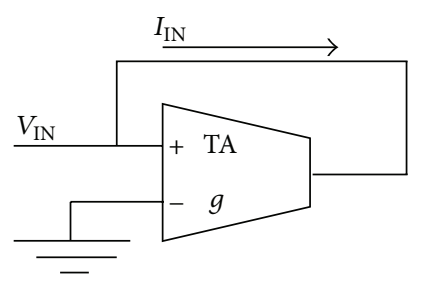

FIGURE 9: TA realization of a grounded resistor.

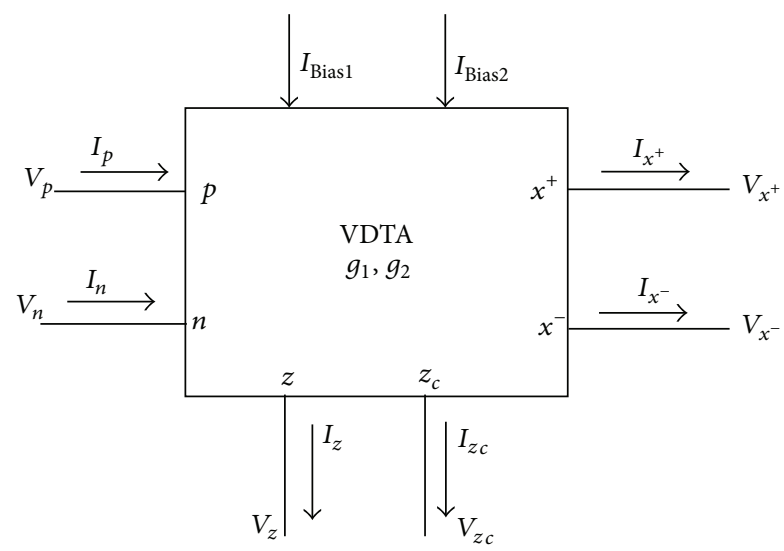

FIGURE 10: Symbol of VDTA.

amplifier is given by (18). The second CDTA block uses two additional TA blocks. It provides both low pass and band pass responses at high output impedance and can be used as Class 0 FAF. The current flowing through $x^{+}, x^{-}, x_{c c}^{+}$, and $x_{c c}^{-}$ is controlled through transconductance $g_{2}$ whereas current flowing through terminals $x_{c}^{+}$and $x_{c}^{-}$is controlled through $g_{3}$ :

$$
A=g_{4} R
$$

The low pass and band pass transfer functions of CDTA based Class 1 FAF are given by (19) and (20), respectively:

$$
\begin{aligned}
& \frac{I_{\mathrm{LP}}}{I_{\mathrm{IN}}}=\frac{g_{1} g_{2}}{C_{1} C_{2} s^{2}+s C_{1} g_{3}+g_{1} g_{2}\left(1+R g_{4}\right)^{2}}, \\
& \frac{I_{\mathrm{BP}}}{I_{\mathrm{IN}}}=\frac{s g_{1} C_{2}}{C_{1} C_{2} s^{2}+s C_{1} g_{3}+g_{1} g_{2}\left(1+R g_{4}\right)^{2}} .
\end{aligned}
$$

The center frequency and quality factor of the CDTA based Class 1 FAF are expressed by (21) and (22), respectively:

$$
\begin{gathered}
f_{0 A}=\frac{1}{2 \pi} \sqrt{\frac{g_{1} g_{2}}{C_{1} C_{2}}}\left(1+g_{4} R\right), \\
Q_{A}=\frac{1}{g_{3}} \sqrt{\frac{g_{1} g_{2} C_{2}}{C_{1}}}\left(1+g_{4} R\right) .
\end{gathered}
$$

The proposed filter uses grounded resistor of value $R$ (= $1 / g$ ) which can easily be implemented using the TA based structure given in Figure 9.

\section{The VDTA Based FAF}

The circuit symbol and the CMOS realization of VDTA [20, 21] are shown in Figures 10 and 11, respectively. The VDTA consists of two transconductance (TC) stages termed as input and output stages. The input differential voltage $\left(V_{p}-V_{n}\right)$ is converted to current $I_{z}$ through TC gain $\left(g_{1}\right)$ of input stage and second stage converts the voltage at $z$ terminal $\left(V_{z}\right)$ to current $\left(I_{x}\right)$ through its TC gain $\left(g_{2}\right)$. The port relations of VDTA can thus be defined by the following matrix:

$$
\left[\begin{array}{c}
I_{z} \\
I_{z_{c}} \\
I_{x^{+}} \\
I_{x^{-}}
\end{array}\right]=\left[\begin{array}{ccc}
g_{1} & -g_{1} & 0 \\
-g_{1} & g_{1} & 0 \\
0 & 0 & g_{2} \\
0 & 0 & -g_{2}
\end{array}\right]\left[\begin{array}{l}
V_{p} \\
V_{n} \\
V_{z}
\end{array}\right] .
$$

The TC $g_{1}$ and TC $g_{2}$ are expressed by (24) which can be adjusted by bias currents $I_{\text {Bias } 1}$ and $I_{\text {Bias2 } 2}$, respectively:

$$
\begin{aligned}
& g_{1}=\sqrt{2 \mu C_{o x}\left(\frac{W}{L}\right)_{1,2} I_{\text {Bias } 1}}, \\
& g_{2}=\sqrt{2 \mu C_{o x}\left(\frac{W}{L}\right)_{5,6} I_{\text {Bias } 2}}
\end{aligned}
$$

4.1. VDTA Based Class 0 FAF. The VDTA based Class 0 FAF employing single VDTA and two grounded capacitors is shown in Figure 12. This circuit configuration is based on second order filter presented in [21]. However, to allow independent control of quality factor and center frequency an additional TA block with transconductance $g_{2}$ is included in VDTA. The current flowing through $z$ terminal is controlled by transconductance $g_{1}$ whereas current flowing through $z_{c}$ terminal is controlled by $g_{2}$. The terminal characteristics of the modified VDTA block are given by (25). The low pass and band pass transfer functions of VDTA based Class 0 FAF are given by (26) and (27), respectively:

$$
\begin{aligned}
{\left[\begin{array}{c}
I_{z} \\
I_{z_{c}} \\
I_{z}^{\prime} \\
I_{z_{c}}^{\prime} \\
I_{x^{+}} \\
I_{x^{-}}
\end{array}\right]=\left[\begin{array}{ccc}
g_{1} & -g_{1} & 0 \\
-g_{1} & g_{1} & 0 \\
g_{2} & -g_{2} & 0 \\
-g_{2} & g_{2} & 0 \\
0 & 0 & g_{3} \\
0 & 0 & -g_{3}
\end{array}\right]\left[\begin{array}{l}
V_{p} \\
V_{n} \\
V_{z}
\end{array}\right], } \\
\frac{I_{\mathrm{LP}}}{I_{\mathrm{IN}}}=\frac{g_{1} g_{3}}{C_{1} C_{2} s^{2}+s C_{2} g_{2}+g_{1} g_{3}}, \\
\frac{I_{\mathrm{BP}}}{I_{\mathrm{IN}}}=\frac{s C_{2} g_{1}}{C_{1} C_{2} s^{2}+s C_{2} g_{2}+g_{1} g_{3}} .
\end{aligned}
$$

The center frequency and quality factor of Class $0 \mathrm{FAF}$ are expressed by (28). The center frequency can be controlled by 


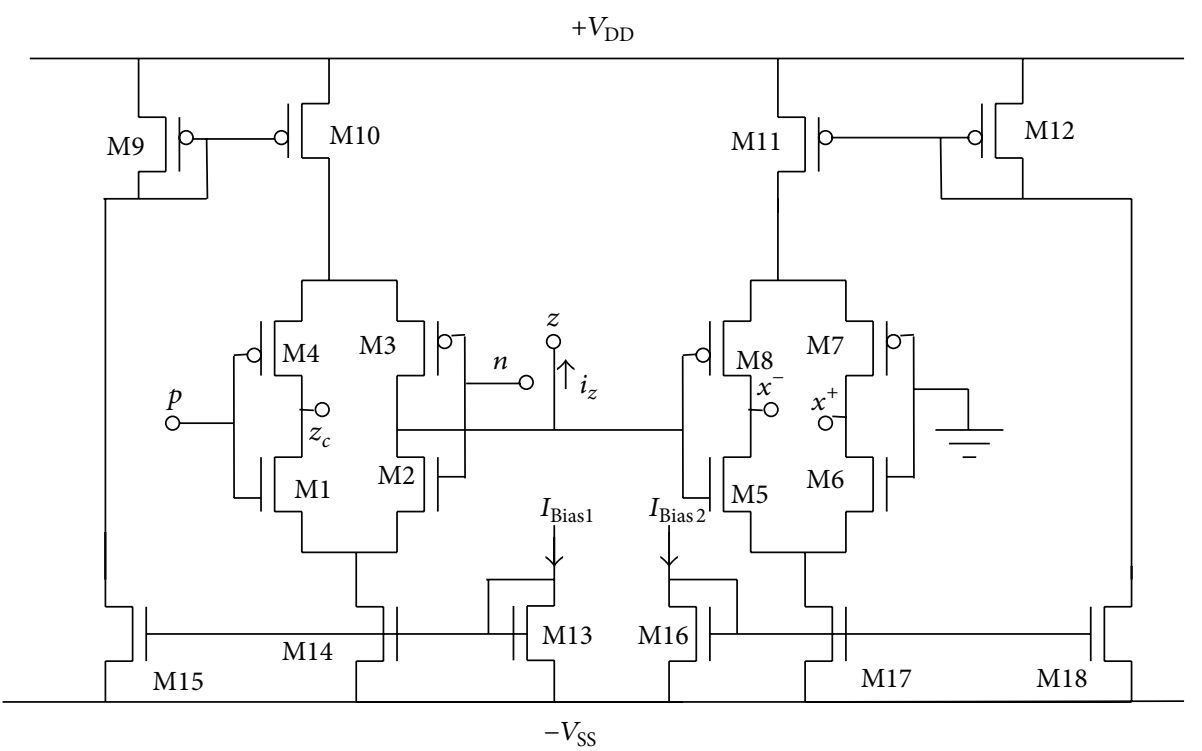

FIGURE 11: CMOS implementation of VDTA [21].

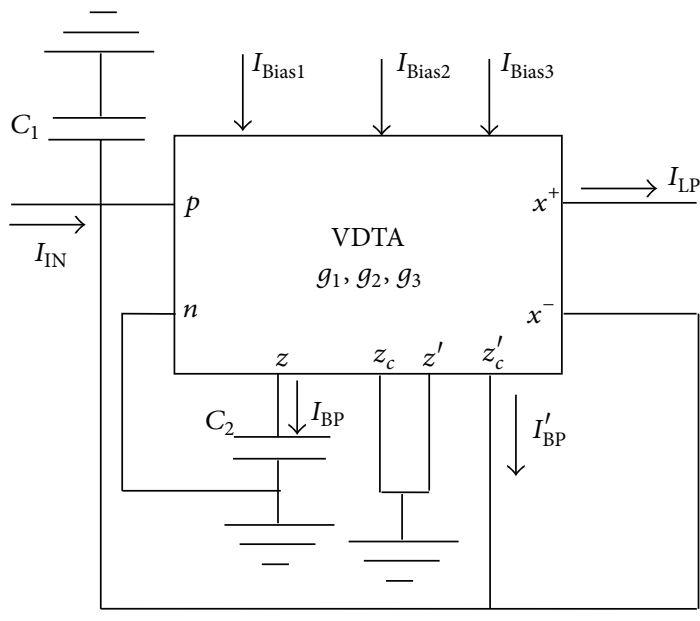

Figure 12: The VDTA based Class 0 FAF.

$I_{\text {Bias1 }}$ and $I_{\text {Bias3 }}$ whereas quality factor can be independently controlled by $I_{\text {Bias } 2}$ :

$$
\begin{gathered}
f_{0}=\frac{1}{2 \pi} \sqrt{\frac{g_{1} g_{3}}{C_{1} C_{2}}}, \\
Q=\frac{1}{g_{2}} \sqrt{\frac{g_{1} g_{3} C_{1}}{C_{2}}} .
\end{gathered}
$$

4.2. VDTA Based Class 1 FAF. The VDTA based Class 1 FAF is shown in Figure 13. It employs two VDTA blocks and two grounded capacitors. The second VDTA block is used as amplifier with tunable gain $A$. The gain $A$ of VDTA based amplifier is given by

$$
A=\frac{g_{4}}{g_{3}}
$$

and can be adjusted by varying $I_{\text {Bias3 }}$ and $I_{\text {Bias4 }}$.
The low pass and band pass transfer functions of VDTA based Class 1 FAF are given by (30) and (31), respectively:

$$
\begin{aligned}
& \frac{I_{\mathrm{LP}}}{I_{\mathrm{IN}}}=\frac{g_{1} g_{3}}{C_{1} C_{2} s^{2}+s C_{2} g_{2}+g_{1} g_{3}\left(1+\left(g_{4} / g_{3}\right)\right)}, \\
& \frac{I_{\mathrm{BP}}}{I_{\mathrm{IN}}}=\frac{s g_{1} C_{2}}{C_{1} C_{2} s^{2}+s C_{2} g_{2}+g_{1} g_{3}\left(1+\left(g_{4} / g_{3}\right)\right)} .
\end{aligned}
$$

The center frequency and quality factor of the CDTA based Class 1 FAF are expressed by (32) and (33), respectively. The center frequency can be independently controlled by varying $I_{\text {Bias2 }}$ without changing center frequency:

$$
\begin{gathered}
f_{0 A}=\frac{1}{2 \pi} \sqrt{\frac{g_{1} g_{3}}{C_{1} C_{2}}}\left(\sqrt{1+\frac{g_{4}}{g_{3}}}\right), \\
Q_{A}=\frac{1}{g_{2}} \sqrt{\frac{g_{1} g_{3} C_{1}}{C_{2}}}\left(\sqrt{1+\frac{g_{4}}{g_{3}}}\right) .
\end{gathered}
$$

4.3. VDTA Based Class 2 FAF. The VDTA based Class 2 FAF is shown in Figure 14 which employs three VDTAs, two grounded capacitors and one grounded resistor. The second VDTA block is used as amplifier with tunable gain $A$. The gain $A$ of VDTA based amplifier is given by (34). The proposed filter uses grounded resistor which can easily be implemented using the TA with transconductance equal to $g_{3}$ based structure given in Figure 9. To realize second order filter, $I_{\text {Bias7 }}$ is set to value of $I_{\text {Bias4 }}$ such that $g_{7}$ is equal to $g_{4}$ and $I_{\text {Bias } 6}$ is set to value such that $g_{6}$ is equal to sum of $g_{3}$ and $g_{4}$; that is, $g_{6}=g_{3}+g_{4}$. Consider

$$
A=\frac{g_{4}}{g_{3}}
$$

which can be adjusted by varying $I_{\text {Bias3 }}$ and $I_{\text {Bias2 } 2}$, thereby making $f_{0 A}$ tunable. 


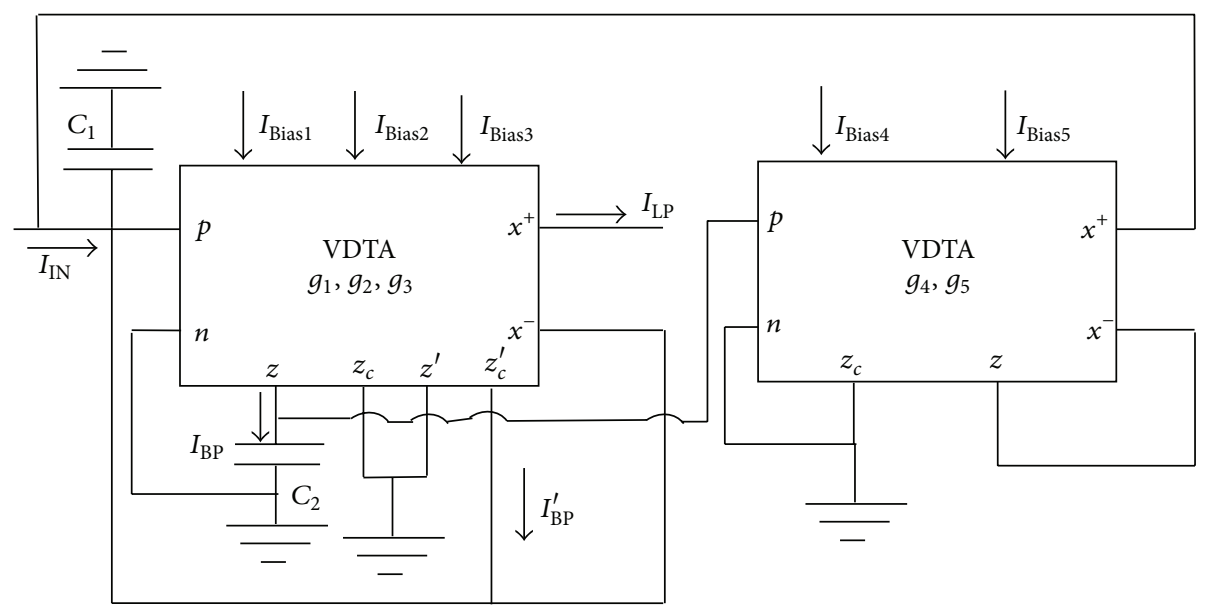

Figure 13: VDTA based Class 1 FAF.

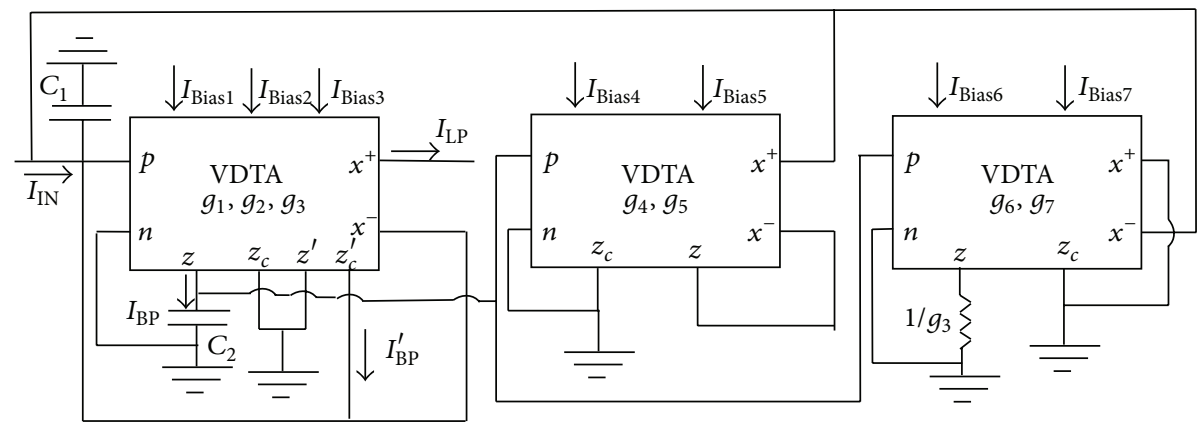

Figure 14: VDTA based Class 2 FAF.

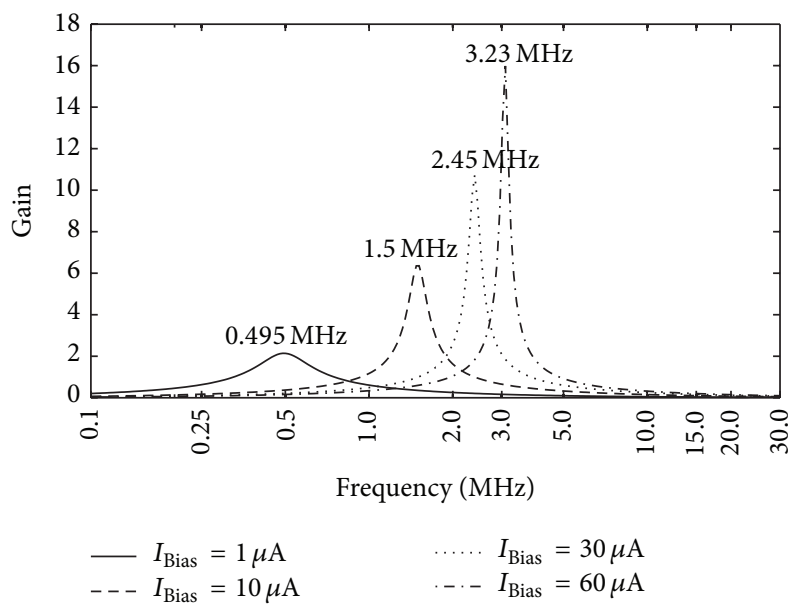

FIGURE 15: Frequency response of CDTA based Class 0 FAF with $I_{\text {Bias1 }}=I_{\text {Bias2 }}=I_{\text {Bias }}$.

The low pass and band pass transfer functions of CDTA based Class 1 FAF are given by (35) and (36), respectively:

$$
\begin{aligned}
& \frac{I_{\mathrm{LP}}}{I_{\mathrm{IN}}}=\frac{g_{1} g_{3}}{C_{1} C_{2} s^{2}+s C_{2} g_{2}+g_{1} g_{3}\left(1+\left(g_{4} / g_{3}\right)\right)^{2}}, \\
& \frac{I_{\mathrm{BP}}}{I_{\mathrm{IN}}}=\frac{s g_{1} C_{2}}{C_{1} C_{2} s^{2}+s C_{2} g_{2}+g_{1} g_{3}\left(1+\left(g_{4} / g_{3}\right)\right)^{2}} .
\end{aligned}
$$

The center frequency and quality factor of the CDTA based Class 1 FAF are expressed by (37) and (38), respectively:

$$
\begin{gathered}
f_{0 A}=\frac{1}{2 \pi} \sqrt{\frac{g_{1} g_{3}}{C_{1} C_{2}}}\left(1+\frac{g_{4}}{g_{3}}\right), \\
Q_{A}=\frac{1}{g_{2}} \sqrt{\frac{g_{1} g_{3} C_{1}}{C_{2}}}\left(1+\frac{g_{4}}{g_{3}}\right) .
\end{gathered}
$$

\section{Nonideal Analysis}

In this section, nonideal analysis of CDTA and VDTA based Class 0 FAF is presented.

5.1. Nonideal Analysis of Class 0 CDTA Based FAF. In practice, the transfer functions (9) and (10) modify due to nonidealities which are classified as tracking errors and parasites. The tracking errors cause current transfer from $p$ and $n$ ports to $z$ port to differ from unity value and are represented by $\alpha_{p}$ and $\alpha_{n}$. There is deviation in transconductance transfer from $z$ to $x^{+}$and $x^{-}$ports which is modeled by $\beta g V_{z}$. The parasites denoted by resistances $R_{p}$ and $R_{n}$ are at $p$ and $n$ terminals; shunt output impedances $(R / / C)$ are present at terminals $z$, $z_{c}, x^{+}$and $x^{-}$, and $x_{c}^{+}$and $x_{c}^{-}$. The effect of the parasites is highly dependent on the topology. A close inspection of the circuit of Figure 6 shows that the parasitic capacitances 


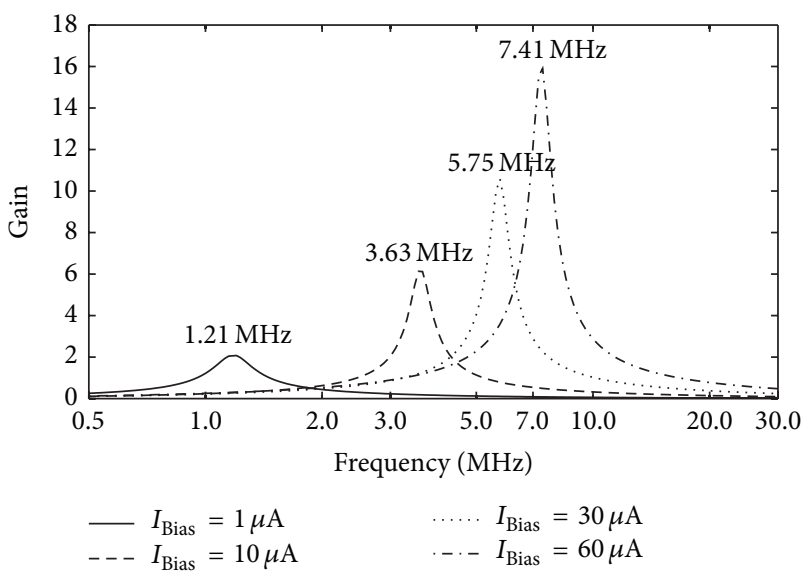

FIGURE 16: Frequency response of CDTA based Class 1 FAF with $I_{\text {Bias } 1}=I_{\text {Bias } 2}=I_{\text {Bias }}$.

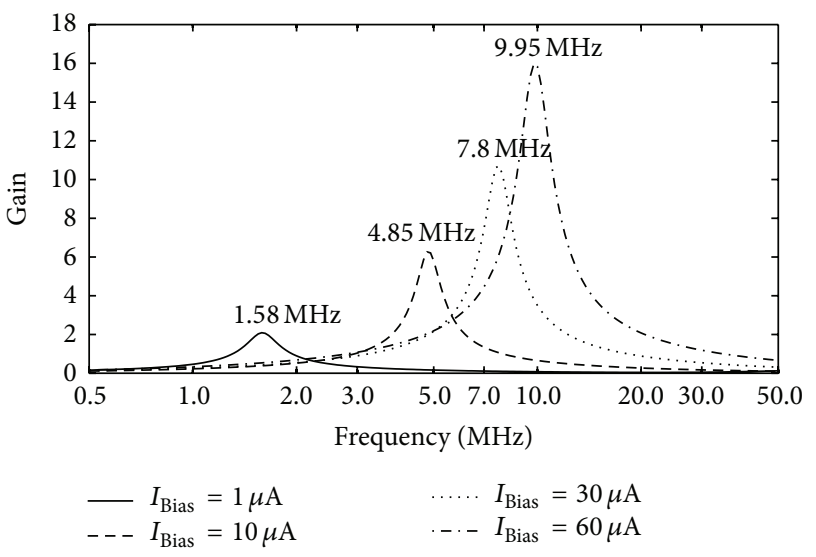

FIGURE 17: Frequency response of CDTA based Class 2 FAF with $I_{\text {Bias } 1}=I_{\text {Bias } 2}=I_{\text {Bias }}$.

present at $z$ terminal can be easily accommodated in external capacitances.

Reanalysis of the proposed circuit (Figure 6) yields the following nonideal transfer functions:

$$
\begin{gathered}
\frac{I_{\mathrm{LP}}}{I_{\mathrm{IN}}}=\frac{\alpha_{n}^{2} \beta^{2} g_{1} g_{2} Q_{1}}{D_{n 1}(s)}, \\
\frac{I_{\mathrm{BP}}}{I_{\mathrm{IN}}}=\frac{\alpha_{n}^{2} \beta g_{1}\left(s C_{2 \mathrm{eq}}+G_{z}\right) Q_{1}}{D_{n 1}(s)},
\end{gathered}
$$

where

$$
\begin{gathered}
D_{n}(s)=P_{1} Q_{1}\left(s C_{1 \mathrm{eq}}+G_{z}\right)\left(s C_{2 \mathrm{eq}}+G_{z}\right) \\
+\alpha_{p} \beta g_{3} P_{1}\left(s C_{1 \mathrm{eq}}+G_{z}\right)+\alpha_{p} \alpha_{n} \beta^{2} g_{1} g_{2}, \\
P_{1}=\left(1+G_{X} R_{n}+s C_{X} R_{n}\right) ; \\
Q_{1}=\left(1+G_{X} R_{p}+s C_{X} R_{p}\right) ; \\
C_{1 \mathrm{eq}}=C_{1}+C_{z} ; \quad C_{2 \mathrm{eq}}=C_{2}+C_{z} ; \\
G_{z}=\frac{1}{R_{Z}} ; \quad G_{X}=\frac{1}{R_{X}} .
\end{gathered}
$$

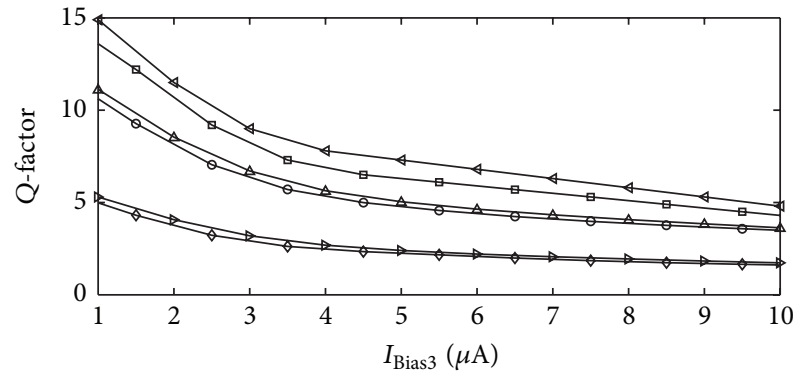

$\rightarrow$ Class 0 FAF analytical response

$\rightarrow$ Class 0 FAF simulated response

$\triangle$ Class 1 FAF analytical response

$\rightarrow$ Class 1 FAF simulated response

$\multimap$ Class 2 FAF analytical response

$\rightarrow$ Class 2 FAF simulated response

FIGURE 18: Electronic Q-factor control of CDTA based FAF.

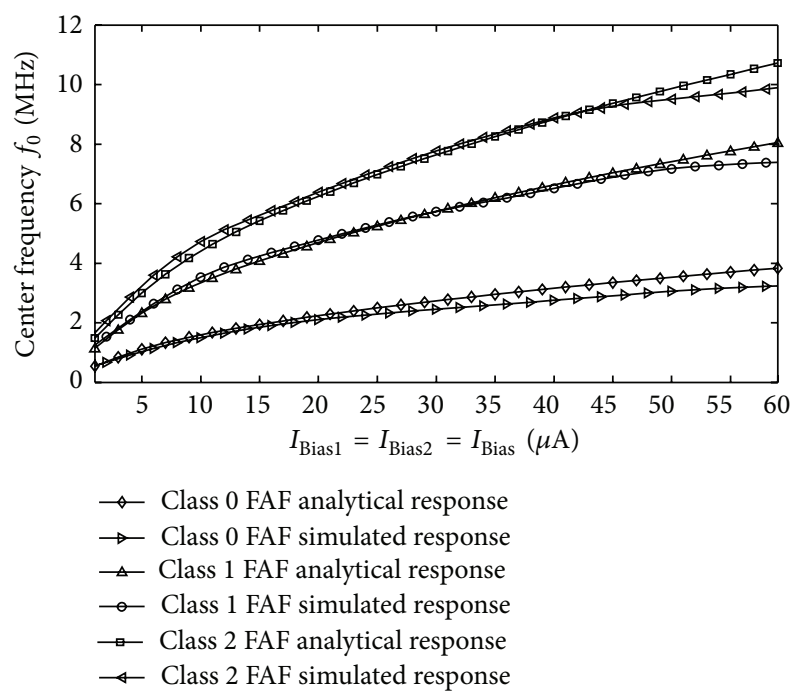

FIGURE 19: Electronic center frequency control of CDTA based FAF.

Choosing operating frequencies below $\min \left(1 / C_{X} R_{p}\right.$, $\left.1 / C_{X} R_{n}\right)$ (as $G_{X} R_{n} \ll 1$ and $G_{X} R_{p} \ll 1$ ) the terms $P_{1}$ and $Q_{1}$ would not affect the transfer function. For frequencies below $\min \left(G_{Z} / C_{1 \text { eq }}, G_{Z} / C_{2 \mathrm{eq}}\right)$, (39c) modifies to

$$
D_{n}(s)=s^{2} C_{1 \mathrm{eq}} C_{2 \mathrm{eq}}+\alpha_{p} \beta g_{3} s C_{1 \mathrm{eq}}+\alpha_{p} \alpha_{n} \beta^{2} g_{1} g_{2}
$$

and transfer functions (39b) and (39c) change to

$$
\begin{gathered}
\frac{I_{\mathrm{LP}}}{I_{\mathrm{IN}}}=\frac{\alpha_{n}^{2} \beta^{2} g_{1} g_{2}}{D_{n}(s)}, \\
\frac{I_{\mathrm{BP}}}{I_{\mathrm{IN}}}=\frac{\alpha_{n}^{2} \beta g_{1} s C_{2 \mathrm{eq}}}{D_{n}(s)} .
\end{gathered}
$$




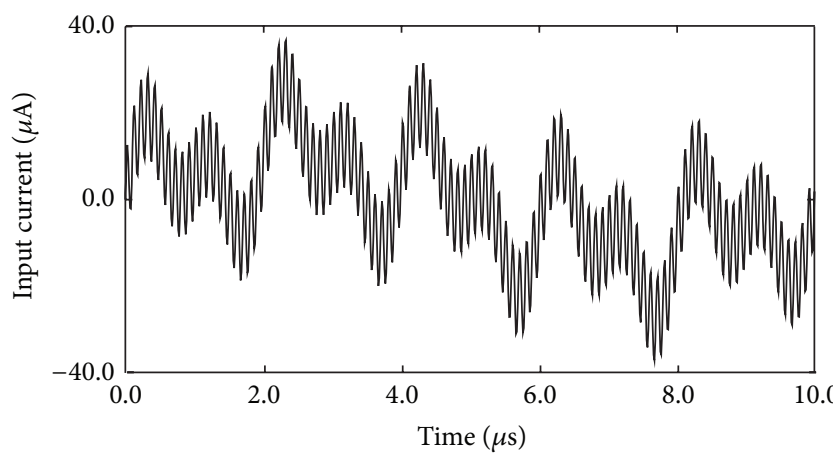

(a)

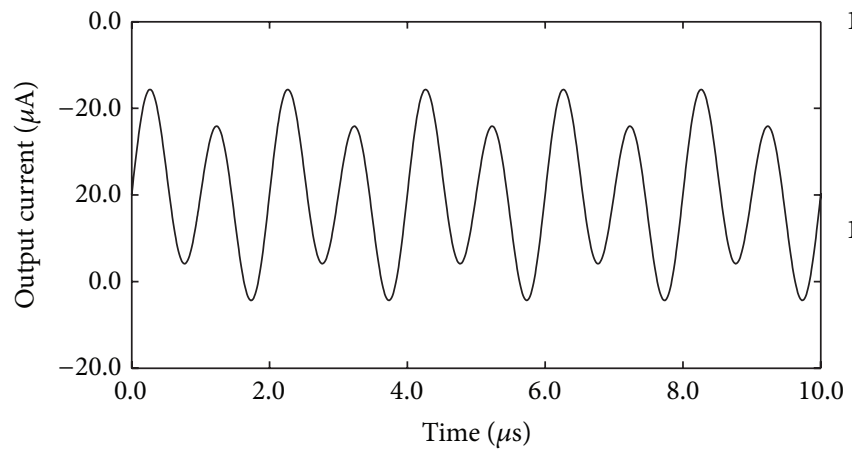

(b)

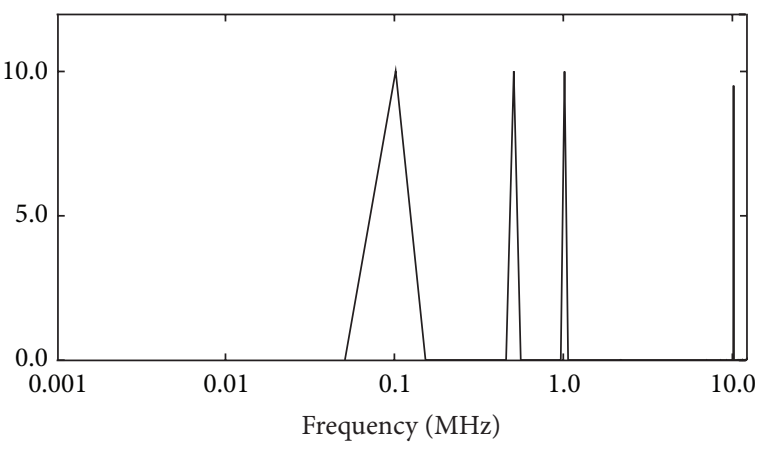

(c)

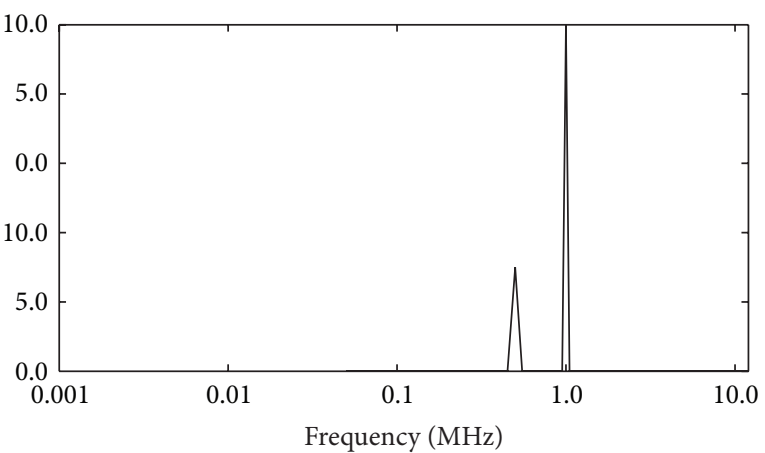

(d)

Figure 20: ((a) and (c)) Input and its frequency spectrum. ((b) and (d)) Output and its frequency spectrum for Class 0 FAF.

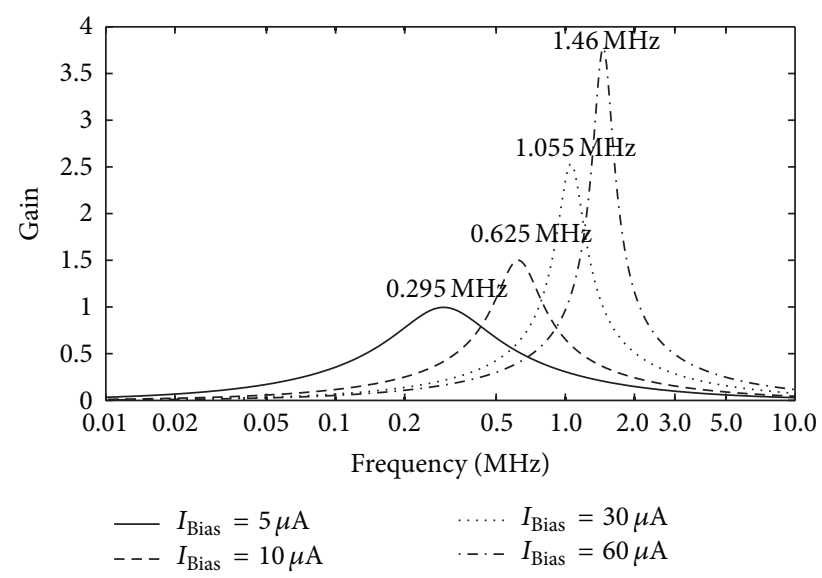

Figure 21: Frequency response of VDTA based Class 0 FAF with $I_{\text {Bias } 1}=I_{\text {Bias } 3}=I_{\text {Bias }}$.

The center frequency, quality factor of Class 0 FAF can be expressed as

$$
\begin{aligned}
& f_{0}=\frac{1}{2 \pi} \sqrt{\frac{\alpha_{n} \alpha_{p} \beta^{2} g_{1} g_{2}}{C_{1 \mathrm{eq}} C_{2 \mathrm{eq}}}}, \\
& Q=\frac{1}{g_{3}} \sqrt{\frac{\alpha_{n} \alpha_{p} g_{1} g_{2} C_{2 \mathrm{eq}}}{C_{1 \mathrm{eq}}}} .
\end{aligned}
$$

It is clear that the transfer functions and filter parameters ((40a), (40b) and (41a), (41b)) deviate from the ideal value in presence of nonidealities. The change can, however, be accommodated by adjusting bias currents.

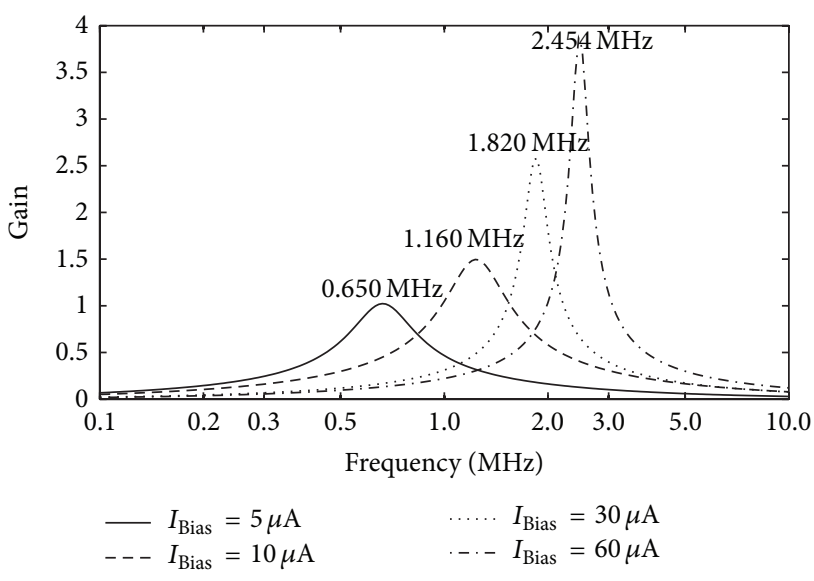

FIGURE 22: Frequency response of VDTA based Class 1 FAF with $I_{\text {Bias } 1}=I_{\text {Bias } 3}=I_{\text {Bias }}$.

5.2. Nonideal Analysis of Class O VDTA Based FAF. Considering the nonideal characteristics of the VDTA, the port relations of current and voltage in (25) can be rewritten as

$$
\left[\begin{array}{c}
I_{z} \\
I_{z_{c}} \\
I_{z}^{\prime} \\
I_{z_{c}}^{\prime} \\
I_{x^{+}} \\
I_{x^{-}}
\end{array}\right]=\left[\begin{array}{ccc}
\beta g_{1} & -\beta g_{1} & 0 \\
-\beta g_{1} & \beta g_{1} & 0 \\
\beta g_{2} & -\beta g_{2} & 0 \\
-\beta g_{2} & \beta g_{2} & 0 \\
0 & 0 & \beta g_{3} \\
0 & 0 & -\beta g_{3}
\end{array}\right]\left[\begin{array}{c}
V_{p} \\
V_{n} \\
V_{z}
\end{array}\right]
$$

where $\beta$ represents the tracking error. Apart from tracking error, the parasites appear as shunt impedances $(R / / C)$ at 


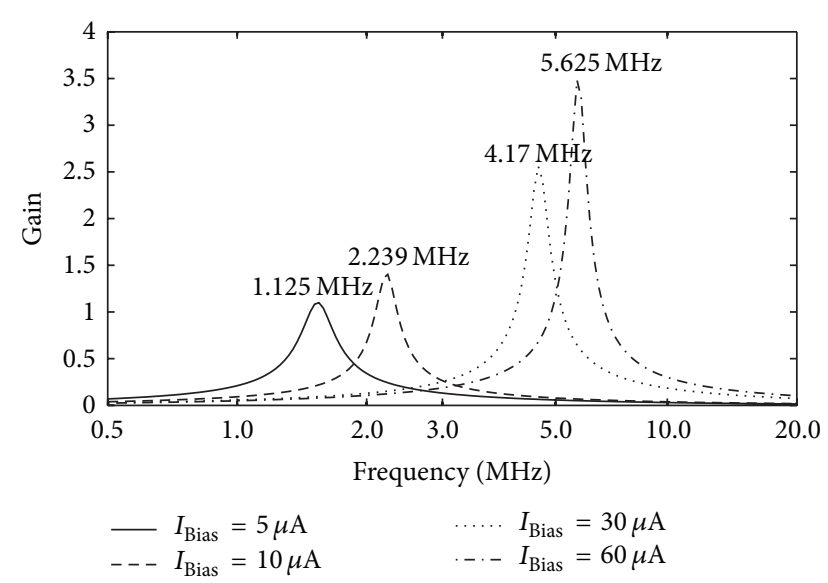

FIGURE 23: Frequency response of VDTA based Class 2 FAF with $I_{\text {Bias } 1}=I_{\text {Bias } 3}=I_{\text {Bias }}$.

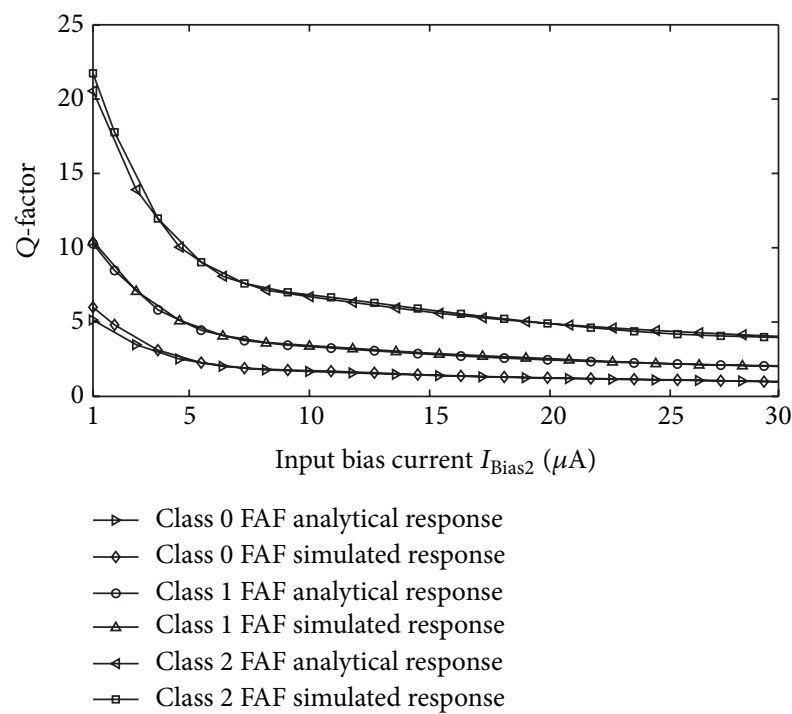

FIGURE 24: Electronic Q-factor control of VDTA based FAF.

ports $p, n, z, z_{c}$, and $x^{+}$denoted by $\left(R_{p} / / C_{p}\right),\left(R_{n} / / C_{n}\right)$, $\left(R_{z} / / C_{z}\right),\left(R_{z} / / C_{z}\right)$, and $\left(R_{x} / / C_{x}\right)$, respectively. The parasitic capacitances present at $p, z, z_{c}$, and $x$ terminal can be easily accommodated in external capacitances.

Reanalysis of the proposed circuit in Figure 12 yields the following nonideal transfer functions of Class 0 VDTA based FAF.

Then

$$
\begin{gathered}
\frac{I_{\mathrm{LP}}}{I_{\mathrm{IN}}}=\frac{g_{1} g_{3} \beta^{2}}{D_{n 2}(s)}, \\
\frac{I_{\mathrm{BP}}}{I_{\mathrm{IN}}}=\frac{\beta g_{1}\left(s C_{2 \mathrm{eq}}+G_{Z}\right)}{D_{n 2}(s)},
\end{gathered}
$$

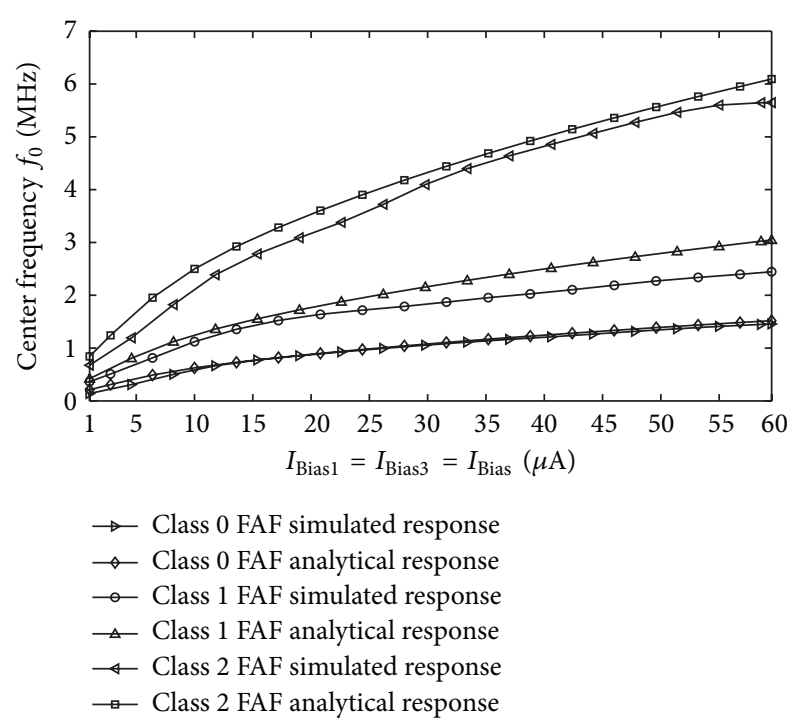

FIGURE 25: Electronic center frequency control of VDTA based FAF.

where

$$
\begin{gathered}
D_{n 2}(s)=\left(s C_{2 \mathrm{eq}}+G_{z}\right)\left(s C_{1 \mathrm{eq}}+G_{x}+G_{z}+G_{p}+\beta g_{2}\right) \\
+\beta^{2} g_{1} g_{3}, \\
C_{1 \mathrm{eq}}=C_{1}+C_{x}+C_{z}+C_{p} ; \quad C_{2 \mathrm{eq}}=C_{2}+C_{z} ; \\
G_{z}=\frac{1}{R_{Z}} ; \quad G_{X}=\frac{1}{R_{X}} ; \\
G_{p}=\frac{1}{R_{p}} .
\end{gathered}
$$

As $G_{x}+G_{z}+G_{p} \ll \beta g_{2},(43 c)$ modifies to

$$
D_{n 2}(s)=\left(s C_{2 \mathrm{eq}}+G_{Z}\right)\left(s C_{1 \mathrm{eq}}+\beta g_{2}\right)+\beta^{2} g_{1} g_{3} .
$$

Choosing operating frequencies below $\min \left(G_{Z} / C_{1 \text { eq }}\right.$, $\left.G_{Z} / C_{2 \mathrm{eq}}\right)(44)$ reduces to

$$
D_{n 2}(s)=s^{2} C_{1 \mathrm{eq}} C_{2 \mathrm{eq}}+s C_{2 \mathrm{eq}} \beta g_{2}+\beta^{2} g_{1} g_{3},
$$

and the transfer function (43b) simplifies to

$$
\begin{gathered}
\frac{I_{\mathrm{LP}}}{I_{\mathrm{IN}}}=\frac{g_{1} g_{3} \beta^{2}}{D_{n 2}(s)}, \\
\frac{I_{\mathrm{BP}}}{I_{\mathrm{IN}}}=\frac{\beta g_{1} s C_{2 \mathrm{eq}}}{D_{n 2}(s)} .
\end{gathered}
$$




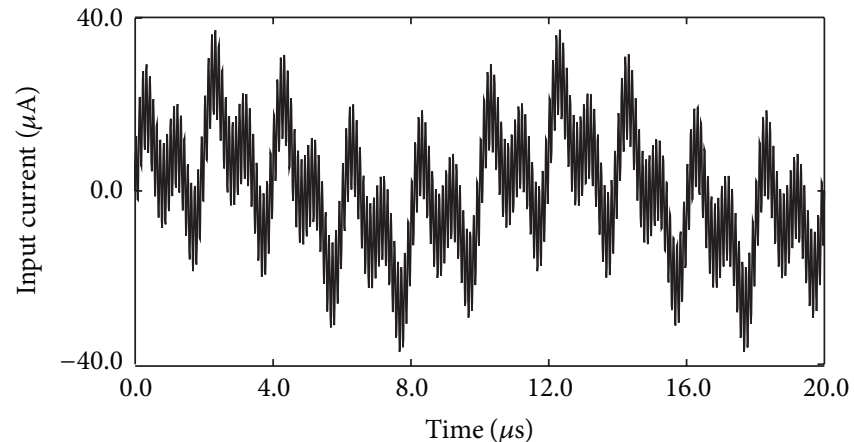

(a)

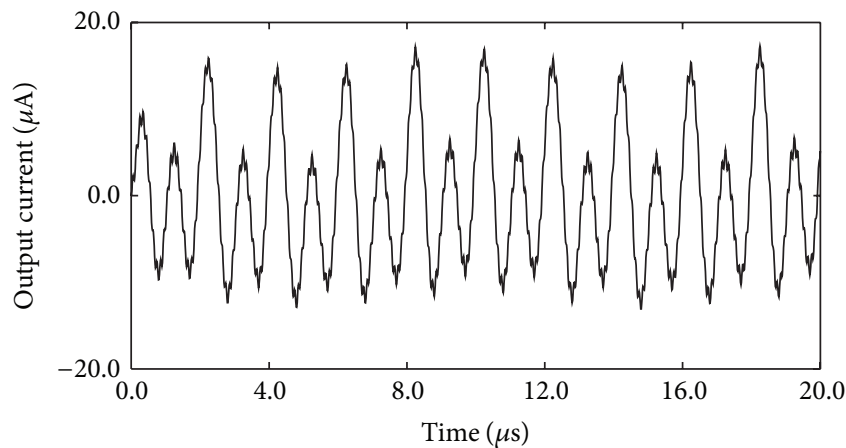

(b)

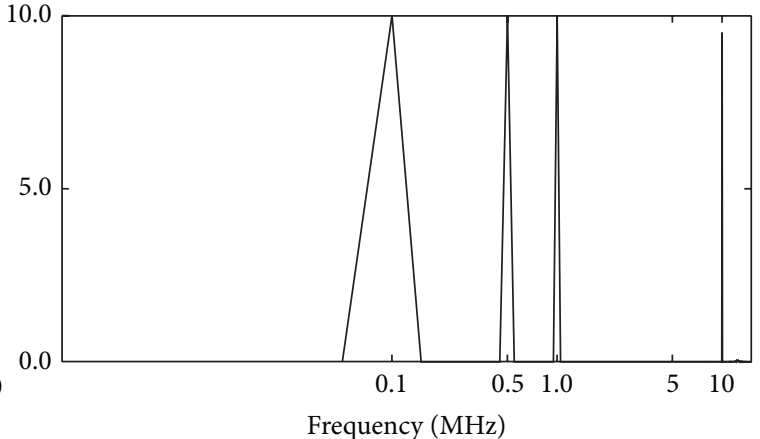

(c)

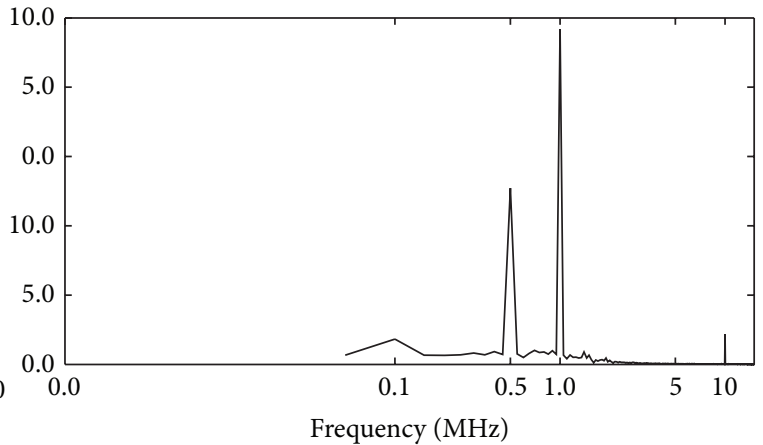

(d)

Figure 26: ((a) and (c)) Input and its frequency spectrum. ((b) and (d)) Output and its frequency spectrum for Class 0 FAF.

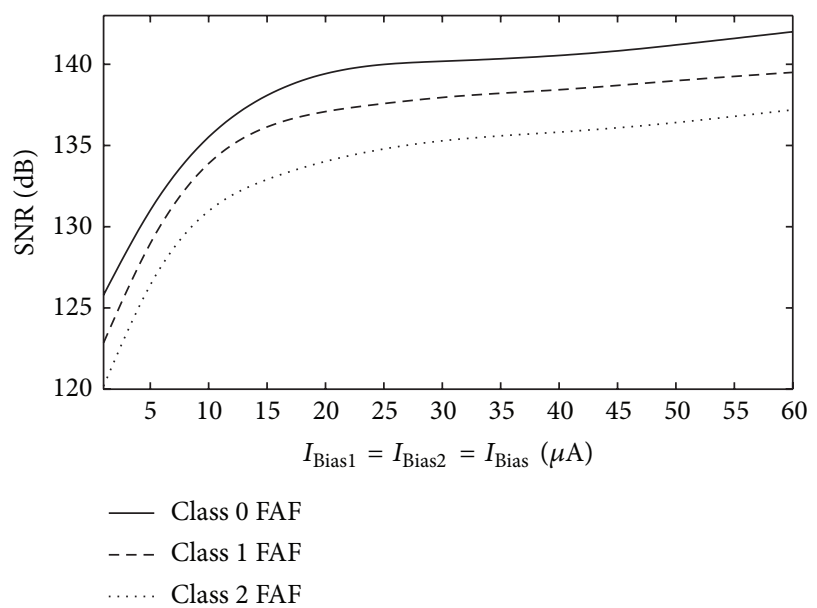

Figure 27: SNR of CDTA based FAF.

And the filter parameters are calculated as

$$
\begin{aligned}
& f_{0}=\frac{1}{2 \pi} \sqrt{\frac{\beta^{2} g_{1} g_{3}}{C_{1 \mathrm{eq}} C_{2 \mathrm{eq}}}}, \\
& Q=\frac{1}{g_{2}} \sqrt{\frac{g_{1} g_{3} C_{2 \mathrm{eq}}}{C_{1 \mathrm{eq}}}} .
\end{aligned}
$$

It is clear that the transfer functions and filter parameters ((46a), (46b) and (47a), (47b)) deviate from the ideal value in presence of nonidealities. The change can, however, be accommodated by adjusting bias currents.

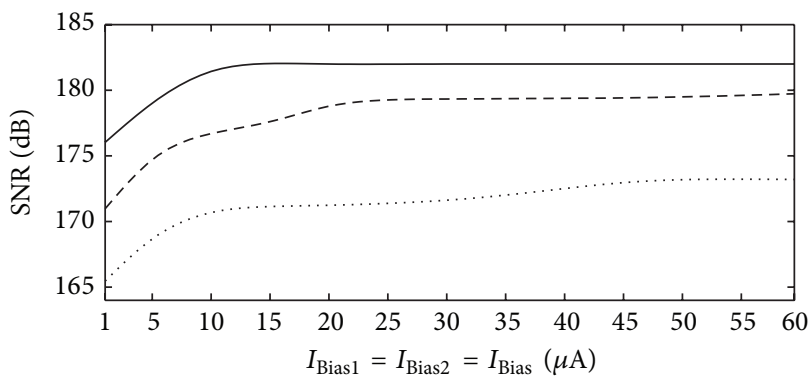

- Class 0 FAF
--- Class 1 FAF
$\ldots .$. Class 2 FAF

FIGURE 28: SNR of VDTA based FAF.

\section{Simulation Results}

In this section, the functionality of the proposed filters has been verified. The SPICE simulations results for CDTA and VDTA based filters have been presented using TSMC $0.25 \mu \mathrm{m}$ CMOS process model parameters and supply voltages of $V_{\mathrm{DD}}=-V_{\mathrm{SS}}=1.8 \mathrm{~V}$.

6.1. Simulation of CDTA Based FAF. The CMOS schematic of Figure 5 is used for verifying CDTA based FAF and the aspect ratios of the MOS transistors are given in Table 1. The additional TA blocks in CDTA providing current ports $\left(x_{C}^{+}, x_{C}^{-}, x_{c c}^{+}\right.$, and $\left.x_{c c}^{-}\right)$use aspect ratios same as that for $x^{+}$ and $x^{-}$. The capacitors $C_{1}$ and $C_{2}$ are chosen as $50 \mathrm{pF}$ each. 
TABLE 1: Aspect ratios of MOS transistors used in CDTA.

\begin{tabular}{lc}
\hline MOSFETs & $W(\mu \mathrm{m}) / L(\mu \mathrm{m})$ \\
\hline Mc26 & $7.0 / 0.7$ \\
Mc1 & $9.8 / 0.7$ \\
Mc2, Mc3, Mc13, Mc16, Mc17 & $10.5 / 0.7$ \\
Mc19, Mc21 & $16.1 / 0.7$ \\
Mc6, Mc20 & $28.0 / 0.7$ \\
Mc8, Mc10, Mc18 & $28.7 / 0.7$ \\
Mc15, Mc12, Mc5 & $35.0 / 0.7$ \\
Mc4, Mc14 & $42.0 / 0.7$ \\
Mc22, Mc23, Mc25 & $56.0 / 0.7$ \\
Mc24 & $58.8 / 0.7$ \\
Mc7, Mc9, Mc11 & $70.0 / 0.7$ \\
\hline
\end{tabular}

The grounded resistor of Figure 7 is realized using TA block. The bias current is set as $0.85 \mu \mathrm{A}$ to realize a resistor of value $10 \mathrm{k} \Omega$. The frequency responses of CDTA based Class 0 , Class 1, and Class 2 FAF topologies are depicted in Figures 15, 16, and 17, respectively. The responses are obtained by varying bias currents $I_{\text {Bias1 }}$ and $I_{\text {Bias2 }}\left(I_{\text {Bias }}=I_{\text {Bias1 }}=I_{\text {Bias2 }}\right)$ to $1 \mu \mathrm{A}, 10 \mu \mathrm{A}, 30 \mu \mathrm{A}$, and $60 \mu \mathrm{A}$ while keeping $I_{\text {Bias3 }}$ and $I_{\text {Bias4 }}$, respectively, at $0.5 \mu \mathrm{A}$ and $10 \mu \mathrm{A}$. It can be clearly noticed that center frequency $f_{0}$ increases on increasing the bias current.

The electronic tunability of quality factor and center frequency for proposed Class 0, Class 1, and Class 2 CDTA based FAF is plotted in Figures 18 and 19, respectively. The analytical and simulated responses describing variation of quality factor are shown in Figure 18 for different values of $I_{\text {Bias3 }}$ while setting $I_{\text {Bias1 }}$ and $I_{\text {Bias2 }}$ to $30 \mu \mathrm{A}$. Figure 19 depicts the analytical and simulated responses for center frequency variation for different values of $I_{\text {Bias1 }}$ and $I_{\text {Bias2 }}\left(I_{\text {Bias1 }}=I_{\text {Bias2 }}\right)$ while setting $I_{\text {Bias3 }}$ to $0.5 \mu \mathrm{A}$. To plot responses for Class 1 and Class 2 CDTA based FAF the bias current $I_{\text {Bias4 }}$ is taken as $10 \mu \mathrm{A}$.

The transient behaviour of proposed CDTA based FAF is also studied by applying input signals of frequencies $100 \mathrm{KHz}$, $500 \mathrm{KHz}, 1 \mathrm{MHz}$, and $10 \mathrm{MHz}$, each having an amplitude of $10 \mu \mathrm{A}$. The responses for Class $0 \mathrm{FAF}$ are obtained by setting bias currents $I_{\text {Bias1 }}$ and $I_{\text {Bias } 2}$ each to $10 \mu \mathrm{A}$ and $I_{\text {Bias3 }}$ to $0.5 \mu \mathrm{A}$. Figure 20 shows the input and output waveforms along with their frequency spectrum for CDTA based Class 0 FAF. It may clearly be noted that the CDTA based Class 0 FAF allows only $1 \mathrm{MHz}$ signal to pass and significantly attenuates signals of frequencies $100 \mathrm{KHz}, 500 \mathrm{KHz}$, and $10 \mathrm{MHz}$. Similar responses for Class 1 and Class 2 FAF were also obtained.

6.2. Simulation of VDTA Based FAF. The CMOS schematic of Figure 12 is used for verifying VDTA based FAF and the aspect ratios of the MOS transistors are given in Table 2. The capacitors $C_{1}$ and $C_{2}$ are taken as $50 \mathrm{pF}$ each. In the realization of Class 2 FAF, the grounded resistor is implemented by TA block. The frequency responses of VDTA based Class 0 , Class 1, and Class 2 FAF topologies are shown in Figures 21,22 , and 23, respectively. The responses are obtained by keeping $I_{\text {Bias2 }}$ to $5 \mu \mathrm{A}$ and setting bias currents $I_{\text {Bias } 1}$ and $I_{\text {Bias3 }}$ $\left(I_{\text {Bias }}=I_{\text {Bias } 1}=I_{\text {Bias3 }}\right)$ to $5 \mu \mathrm{A}, 10 \mu \mathrm{A}, 30 \mu \mathrm{A}$, and $60 \mu \mathrm{A}$. In
TABLE 2: Aspect ratios of MOS transistors used in VDTA.

\begin{tabular}{lc}
\hline MOSFETs & $W(\mu \mathrm{m}) / L(\mu \mathrm{m})$ \\
\hline M1, M2, M5, M6 & $16.1 / 0.7$ \\
M3, M4, M7, M8 & $28 / 0.7$ \\
M9-M12 & $21 / 0.7$ \\
M13-M16 & $7 / 0.7$ \\
M14, M15, M17, M18 & $8.5 / 0.7$ \\
\hline
\end{tabular}

realization of Class $1 \mathrm{FAF}, I_{\text {Bias } 4}$ is set to obtain $g_{4} / g_{3}=3$ while keeping $I_{\text {Bias } 5}$ equal to $I_{\text {Bias } 1}$. In realization of Class $2 \mathrm{FAF}, I_{\text {Bias } 6}$ is selected such that $g_{6}=g_{3}+g_{4}$ while $I_{\text {Bias } 7}$ is equal to $I_{\text {Bias } 4}$.

The electronic tunability of quality factor and center frequency for proposed Class 0, Class 1, and Class 2 VDTA based FAF is plotted in Figures 24 and 25, respectively. The analytical and simulated responses describing variation of quality factor are shown in Figure 24 for different values of $I_{\text {Bias2 }}$ while setting $I_{\text {Bias1 }}$ and $I_{\text {Bias3 }}$ to $30 \mu \mathrm{A}$ in Class 0 . To plot responses for Class $1, I_{\text {Bias } 4}$ is set to get $g_{4} / g_{3}=3$ and $I_{\text {Bias } 5}$ is set equal to $I_{\text {Biasl }}$. Class 2 FAF responses are plotted by selecting $I_{\text {Bias6 }}$ to obtain $g_{6}=g_{3}+g_{4}$ while $I_{\text {Bias7 }}$ is equal to $I_{\text {Bias4 }}$. Figure 25 depicts the analytical and simulated responses for center frequency variation for different values of $I_{\text {Bias1 }}$ and $I_{\text {Bias3 }}\left(I_{\text {Bias1 }}=I_{\text {Bias3 }}\right)$ and keeping $I_{\text {Bias2 }}$ to $5 \mu \mathrm{A}$. To plot responses for Class $1, I_{\mathrm{Bias} 4}$ is set to a value in such a manner that $g_{4} / g_{3}=3$ whereas $I_{\text {Bias } 5}$ is set equal to $I_{\text {Bias } 1}$. The Class 2 FAF responses are plotted by setting $I_{\text {Bias6 }}$ to value such that $g_{6}=g_{3}+g_{4}$ whereas $I_{\text {Bias7 }}$ is equal to $I_{\text {Bias4 } 4}$.

The transient behaviour of proposed agile filter is also studied by applying input signals of frequencies $100 \mathrm{KHz}$, $500 \mathrm{KHz}, 1 \mathrm{MHz}$, and $10 \mathrm{MHz}$, each having an amplitude of $10 \mu \mathrm{A}$. The responses for Class $0 \mathrm{FAF}$ are obtained by setting bias currents $I_{\text {Bias } 1}, I_{\text {Bias2 } 2}$, and $I_{\text {Bias3 }}$ each to $30 \mu \mathrm{A}$. The input and output waveforms along with their frequency spectrum for VDTA based Class 0 FAF are shown in Figure 26. It may clearly be noted that the VDTA based Class 0 FAF allows only $1 \mathrm{MHz}$ signal to pass, partially attenuates signal of frequency $500 \mathrm{KHz}$, and significantly attenuates signals of frequencies $100 \mathrm{KHz}$ and $10 \mathrm{MHz}$. Similar responses for Class 1 and Class 2 FAF are also obtained.

\section{Performance Evaluation}

The performance of proposed CDTA and VDTA based FAF circuits is studied in terms of power dissipation, output noise voltage, and SNR. The overall performance characteristics are summarized in Table 3. Figures 27 and 28 depict the signal to noise ratio (SNR) for the proposed CDTA and VDTA based filter topologies for Class 0 , Class 1 , and Class 2, respectively. The VDTA based FAF proved to be optimum concerning the power dissipation and signal to noise ratio. The maximum output noise voltage is better in VDTA based FAF.

\section{Conclusion}

In this paper CDTA and VDTA based frequency agile filters are presented. The proposed FAF configurations employ 


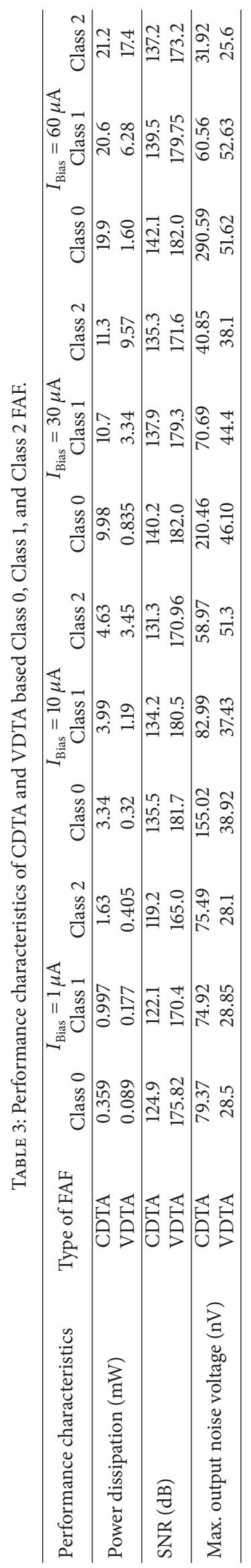


grounded passive components and are suitable for integration. The filter configurations are designed in such a way that quality factor can be independently controlled without changing the center frequency. The simulation results are included to demonstrate the workability of the circuits. The performance of the proposed FAF is evaluated in terms of power dissipation, SNR, and noise performance. The VDTA based FAF proved to be optimum concerning the power dissipation and signal to noise ratio.

\section{Conflict of Interests}

The authors declare that there is no conflict of interests regarding the publication of this paper.

\section{References}

[1] P. I. Mak, U. Seng-Pan, and R. P. Martins, Analog-Baseband Architecture and Circuits for Multistandard and Low Voltage Wireless Transceivers, Analog Integrated Circuits and Signal Processing, 2007.

[2] Y. Lakys and A. Fabre, "Multistandard transceivers: state of the art and a new versatile implementation for fully active frequency agile filters," Analog Integrated Circuits and Signal Processing, vol. 74, no. 1, pp. 63-78, 2013.

[3] Y. Lakys and A. Fabre, "A fully active frequency agile filter for multistandard transceivers," in Proceedings of the International Conference on Applied Electronics (AE '11), pp. 1-7, September 2011.

[4] S. Kaehlert, D. Bormann, T. D. Werth, M.-D. Wei, L. Liao, and S. Heinen, "Design of frequency agile filters in RF frontend circuits," in Proceedings of the IEEE Radio and Wireless Symposium (RWS '12), pp. 13-16, Santa Clara, Calif, USA, January 2012.

[5] A. J. X. Chen, Y. Wu, J. Hodiak, and P. K. L. Yu, "Frequency agile digitally tunable microwave photonic filter," in Proceedings of the International Topical Meeting on Microwave Photonics (MWP '03), pp. 89-92, September 2003.

[6] G. Subramanyam, F. W. van Keuls, and F. A. Miranda, "A Kband-frequency agile microstrip bandpass filter using a thinfilm hts/ferroelectric/dielectric multilayer configuration," IEEE Transactions on Microwave Theory and Techniques, vol. 48, no. 4, pp. 525-530, 2000.

[7] H. Chandrahalim, S. A. Bhave, R. G. Polcawich, J. Pulskamp, and R. Kaul, " $\mathrm{A} \mathrm{Pb}\left(\mathrm{Zr}_{0.55} \mathrm{Ti}_{0.45}\right) \mathrm{O}_{3}$-transduced fully differential mechanically coupled frequency agile filter," IEEE Electron Device Letters, vol. 30, no. 12, pp. 1296-1298, 2009.

[8] M. W. Wyville, R. C. Smiley, and J. S. Wight, "Frequency agile RF filter for interference attenuation," in Proceedings of the 6th IEEE Radio and Wireless Week (RWW '12), pp. 399-402, Santa Clara, Calif, USA, January 2012.

[9] H. H. Sigmarsson, J. Lee, D. Peroulis, and W. J. Chappell, "Reconfigurable-order bandpass filter for frequency agile systems," in Proceedings of the IEEE MTT-S International Microwave Symposium (MTT '10), pp. 1756-1759, Anaheim, Calif, USA, May 2010.

[10] Y. Lakys, B. Godara, and A. Fabre, "Cognitive and encrypted communications, part 2: a new approach to active frequencyagile filters and validation results for an agile bandpass topology in SiGe-BiCMOS," in Proceedings of the 6th International Conference on Electrical and Electronics Engineering (ELECO '09), pp. II16-II29, November 2009.
[11] V. Biolkova and D. Biolek, "Shadow filters for orthogonal modification of characteristic frequency and bandwidth," Electronics Letters, vol. 46, no. 12, pp. 830-831, 2010.

[12] Y. Lakys and A. Fabre, "Shadow filters-new family of secondorder filters," Electronics Letters, vol. 46, no. 4, pp. 276-277, 2010.

[13] D. Biolek, "CDTA-building block for current-mode analog signal processing," in Proceedings of the European Conference on Circuit Theory and Design (ECCTD '03), vol. III, pp. 397-400, Krakow, Poland, 2003.

[14] D. Biolek, V. Biolkova, and Z. Kolka, "Current-mode biquad employing single CDTA," Indian Journal of Pure and Applied Physics, vol. 47, no. 7, pp. 535-537, 2009.

[15] M. Kumngern, P. Phatsornsiri, and K. Dejhan, "Four inputs and one output current-mode multifunction filter using CDTAs and all-grounded passive components," in Proceedings of the 10th International Conference on ICT and Knowledge Engineering, ICT and Knowledge Engineering, pp. 59-62, Bangkok, Thailand, November 2012.

[16] S. K. Pandey, A. P. Singh, M. Kumar, S. Dubey, and P. Tyagi, "A current mode second order filter using dual output CDTA," International Journal of Computer Science \& Communication Networks, pp. 210-213, 2012.

[17] F. Kacar and H. Kuntman, "A new cmos current differencing transconductance amplifier (CDTA) and its biquad filter application," in Proceedings of the IEEE (EUROCON '09), pp. 189-196, St Petersburg, Russia, May 2009.

[18] D. Biolek, E. Hancioglu, and A. Ü. Keskin, "High-performance current differencing transconductance amplifier and its application in precision current-mode rectification," International Journal of Electronics and Communications, vol. 62, no. 2, pp. 92-96, 2008.

[19] A. Uygur, H. Kuntman, and A. Zeki, "Multi-input multioutput CDTA-based KHN filter," in Proceedings of the IEEE International Microwave Symposium Digest, pp. 1756-1759, 2010.

[20] W. Chiu, S. I. Liu, H. W. Tsao, and J. J. Chen, "CMOS differential difference current conveyor and their applications," IEE Proceedings-Circuits Devices Systems, vol. 143, no. 2, pp. 9196, 1996.

[21] Z. Wang, "2-MOSFET transresistor with extremely low distortion for output reaching supply voltages," Electronics Letters, vol. 26, no. 13, pp. 951-952, 1990.

[22] D. Biolek, M. Shaktour, V. Biolkova, and Z. Kolka, "Currentinput current-output universal biquad employing two bulkdriven VDTAs," in Proceedings of the 4th International Congress on Ultra Modern Telecommunications and Control Systems (ICUMT '12), pp. 484-489, St. Petersburg, Russia, October 2012.

[23] J. Satansupa and W. Tangsrirata, "Single VDTA based current mode electronically tunable multifunction filter," in Proceedings of the 4th International Science, Social Science, Engineering and Energy Conference (ISEEC '12), pp. 1-8, 2012.

[24] P. Phatsornsiri, P. Lamun, M. Kumngern, and U. Torteanchai, "Current-mode third-order quadrature oscillator using VDTAs and grounded capacitors," in Proceedings of the 4th Joint International Conference on Information and Communication Technology, Electronic and Electrical Engineering (JICTEE '14), pp. 1-4, IEEE, Chiang Rai, Thailand, March 2014.

[25] T. Pourak, P. Suwanjan, W. Jaikla, and S. Maneewan, "Simple quadrature sinusoidal oscillator with orthogonal control using single active element," in Proceedings of the International Conference on Signal Processing and Integrated Networks (SPIN '14), pp. 1-4, 2014. 
[26] M. Srivastava, D. Prasad, and D. R. Bhaskar, "New Parallel R-L impedance using single VDTA and its high pass filter application," in Proceedings of the International Conference on Signal Processing and Integrated Networks (SPIN '14), pp. 535537, 2014.

[27] K. Chumwangwapee, W. Jaikla, W. Sunthonkanokpong, W. Jaikhang, S. Maneewan, and B. Sreewirote, "High input impedance mixed-mode biquad filter with orthogonal tune of natural frequency and quality factor," in Proceedings of the 4th Joint International Conference on Information and Communication Technology, Electronic and Electrical Engineering (JICTEE '14), pp. 1-4, Chiang Rai, Thailand, March 2014. 

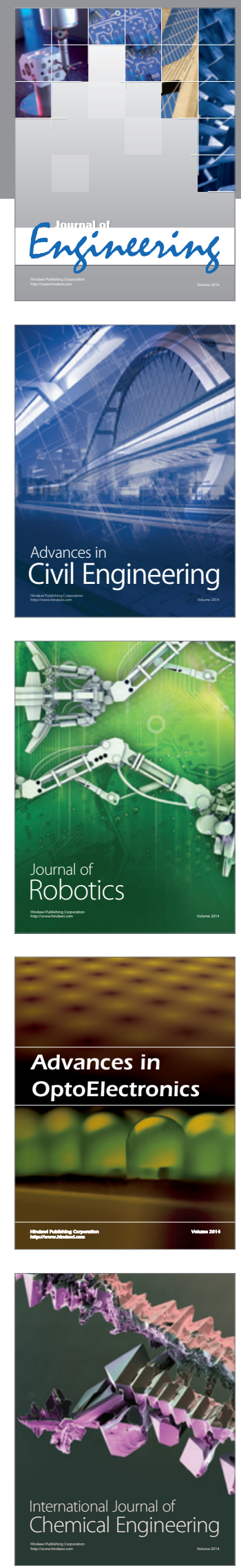

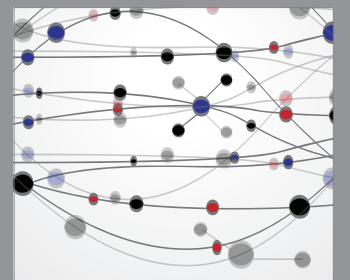

The Scientific World Journal
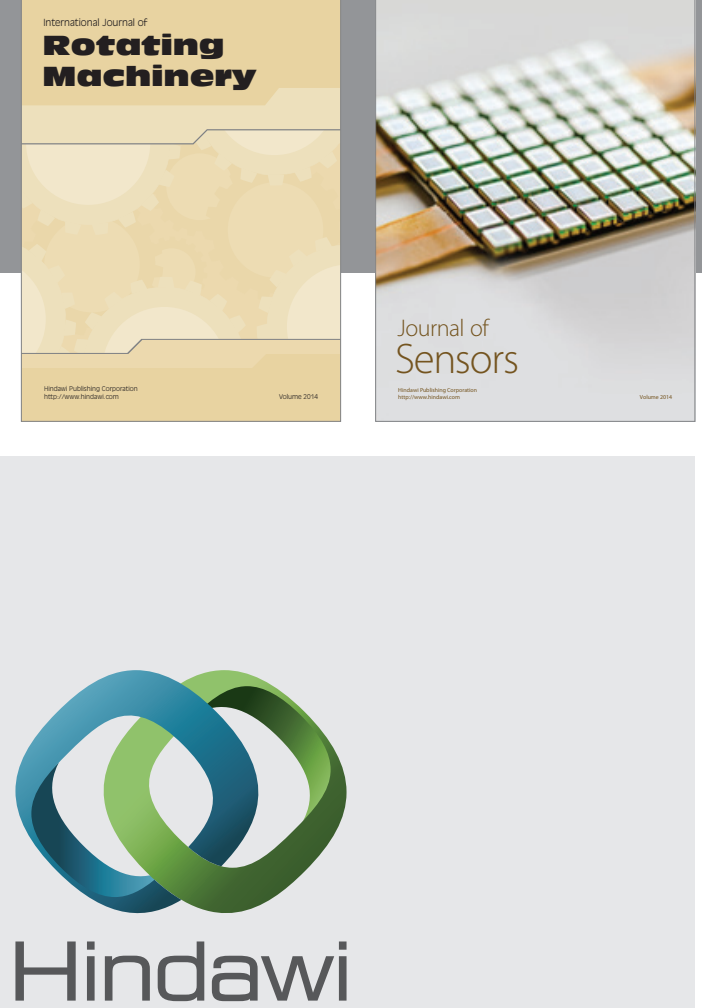

Submit your manuscripts at http://www.hindawi.com
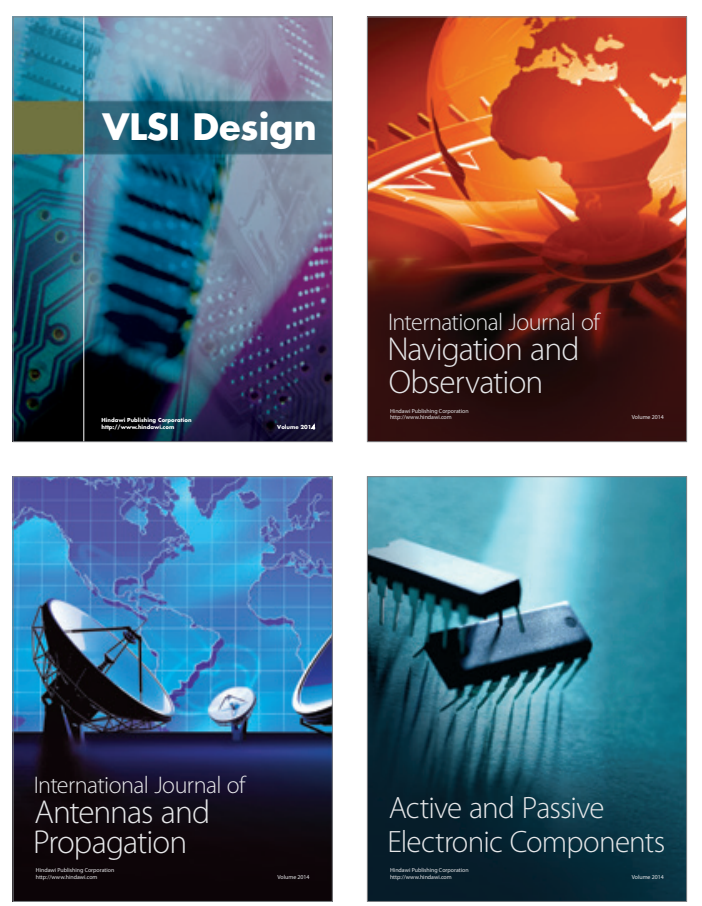
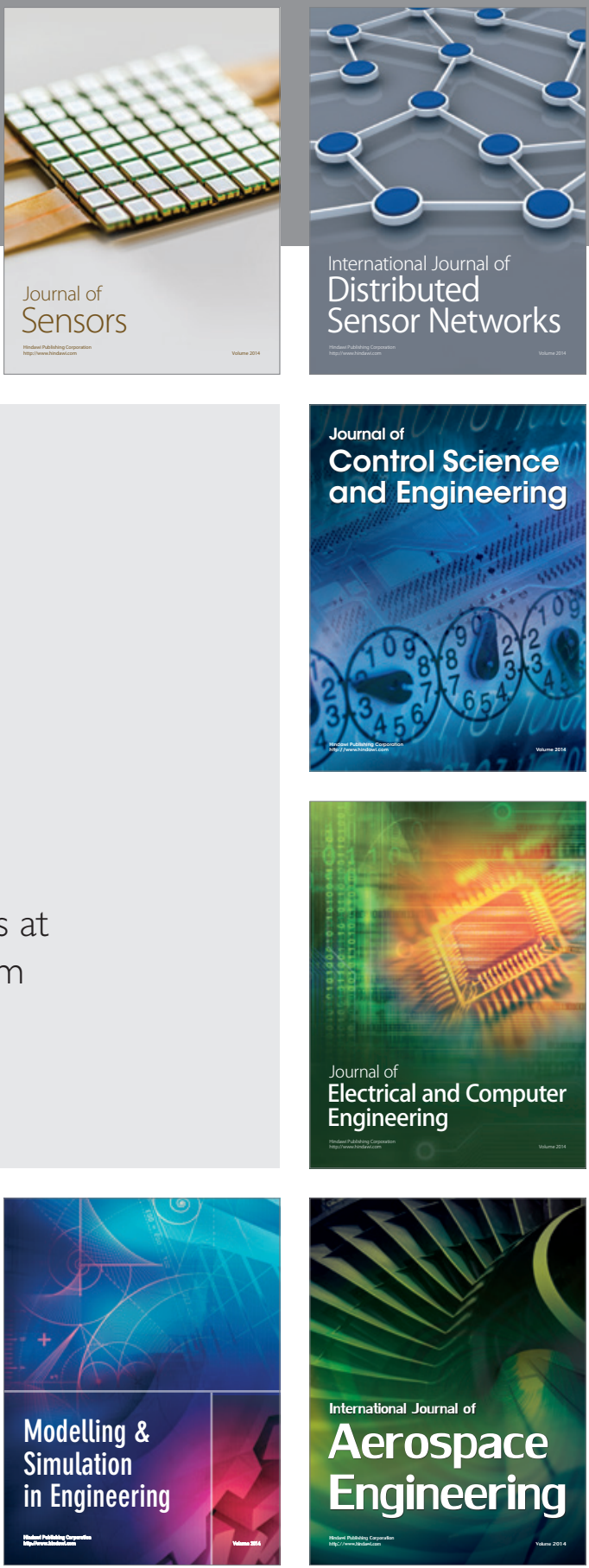

Journal of

Control Science

and Engineering
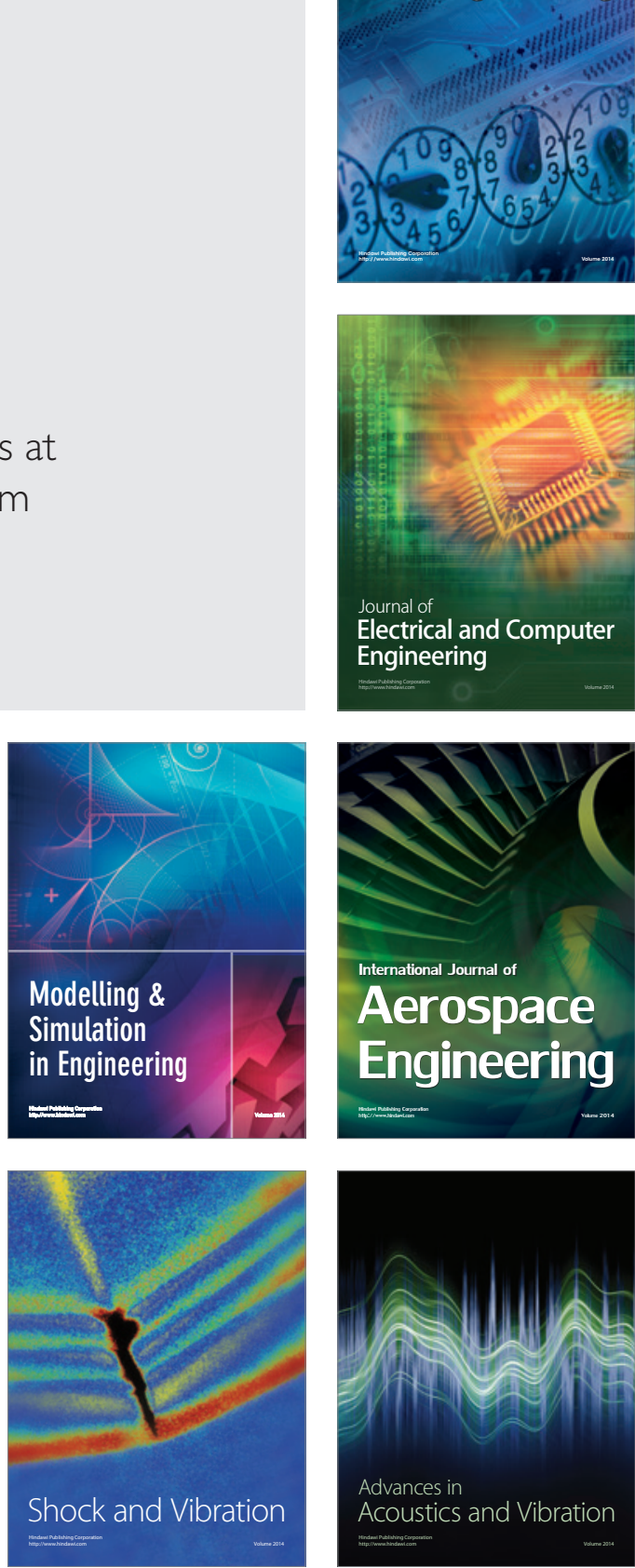